\title{
HEAT-CONDUCTING VISCOUS FLUIDS OVER POROUS MEDIA*
}

\author{
LUISA CONSIGLIERI ${ }^{\dagger}$
}

\begin{abstract}
A new model is introduced for describing the heat-conducting viscous fluids over porous media. The innovative features of the presented model are the nonlinear character given by temperature dependence of the physical parameters such as the viscosities, the permeability, and complementary the thermal conductivity and thermal expansion. The flow velocities are small (for steady processes) and mainly driven by the pressure gradient in porous media such that the Stokes-Darcy system is completed by the energy equation with the heat flux given by the Fourier law. The existence of solutions is established for the Stokes-Darcy-Fourier system either with the Beavers-Joseph-Saffman or Beavers-Joseph interface boundary conditions. Both problems are solved by means a fixed point procedure and Lagrange multiplier approach.
\end{abstract}

Key words. Stokes-Darcy equations, Beavers-Joseph boundary condition, Fourier law.

AMS subject classifications. 76S05, 35J88, 86A60.

\section{Introduction}

The isothermal problem of slow motion over porous media is governed by different partial differential equations in the two regions, typically Stokes and Darcy equations, respectively, giving rise to a coupled problem of heterogeneous type. The mathematical formulation based on the Stokes-Darcy model is completed by considering suitable interface conditions that relate the different mathematical unknowns (e.g. fluid velocity and pressure) from either subdomains across the separating interface.

From the applicative side, this situation is relevant to several fields. From hydrological environmental applications, for instance the karst aquifer among groundwater systems, the free flow is confined in the underground conduit, denoted by $\Omega_{f}$, which connects a sinkhole to a spring. Surrounding the conduit are porous media such as soil, gravel, sand, etc. The porous media as a whole is regarded as the matrix that holds water. From bio-engineering applications, we can refer to the main blood stream in $\Omega_{f}$ through a porous membrane, here represented by $\Omega_{p}$.

Previous studies have developed numerical techniques for Stokes-Darcy systems [2, 3, 10, 13, 29, 33, 34. In [2, the authors analyzed the flow of an incompressible fluid in a $2 \mathrm{D}$ medium composed of a porous material with relatively large cavities, called vugs in the geological literature, which occur ubiquitously throughout, for example, most carbonate rock formations.

New approaches have been proposed over the past few years to model and understand the non isothermal fluid flow through a porous medium (see [9] and references therein). Indeed, studies on flow and heat transfer through porous media cover a broad range of different fields and engineering applications such as ground-water hydrology, petroleum reservoir and geothermal operations, electronic cooling, building thermal insulation, drying technology, multiphase catalytic reactors, and others.

A saltless solar pond consists of continuously injecting cool make-up water from the top, while warm water is withdrawn at the same rate from the bottom surface for distribution. Thus, a gentle downward flow of water is created in the direction of increasing temperature gradient [24].

\footnotetext{
*Received: August 14, 2011; accepted: Oct 24, 2011. Communicated by Shi Jin.

${ }^{\dagger}$ Independent Research Professor, Painel da Lameira Cx. 8, 7300-405 Portalegre, Portugal (lconsiglieri@gmail.com).http://sites.google.com/site/luisaconsiglieri
} 
In the case of carbonate platforms, seawater entering the platform flank is supersaturated with respect to dolomite, and the water reacts with calcite along flow paths within the platform. Geothermal convection, which drives fluids through deep and warm areas of a carbonate platform, arises because geothermal gradients create a density contrast between cold seawater and warm fluids within carbonate platforms. Warm fluids in the center of the platform rise and are replaced by cool seawater, which is in turn heated and rises [36.

In [18 the authors investigated steam flows through a porous pipe and cooling liquid flows through the annular region between a solid outer pipe and the porous inner pipe. The steam condenses onto the inner wall of the porous material because the cooling liquid keeps the temperature of the porous wall below that of the steam's saturation temperature. A small pressure drop is maintained between the steam and the cooling flow in order to drive the condensate through the porous material to the annular region, where it mixes with the cooling liquid and is swept away to a heat exchanger.

Due to the presence of the thermal effects, the physical parameters such as permeability, thermal conductivity, and fluid viscosities are nonlinear and temperature dependent. For instance, water has a viscosity of $0.89 \mathrm{cP}$ at $25^{\circ} \mathrm{C}$, and $1 \mathrm{cP}$ at $20^{\circ} \mathrm{C}$. Other dependencies, like the thermal conductivity on the porosity and velocity, under local thermal non-equilibrium conditions [1, are out of the scope of this work.

In the present paper, fully coupled models are set for the non-isothermal interaction between fluid flow in the porous medium and purely fluid regions. The porous medium has permeability which is heterogeneous and anisotropic.

We deal with the existence of the solution for a low Reynold's number flow over porous media. The bidomain Stokes-Darcy problem consists of an elliptic system, namely a Stokes equation on part of the domain coupled to a Darcy equation on the rest of the domain. Moreover, the thermoflow problem is usually described by a coupled system, known as coupled fluid-energy system [12], constituted by the motion equations for incompressible viscous fluids and the energy equation when the heat transfer is taken into account. In this work, we allow the incompressible condition to vary, so that it corresponds to reality. Indeed, heat-conducting viscous flows are thermally compressible (see [25, 31] and the references therein) and include the so-called weakly compressible flow [32, p. 278]. Notwithstanding, we deal with the Newtonian fluid (linearly viscous fluid). The heat-conducting behavior on mechanically incompressible non-Newtonian fluids, as in [11, overlying porous media will be handled in a forthcoming work.

The proposed solutions are constructed by taking the soil behavior into account and ensuring either continuities on the porous medium/fluid interface, namely the Beavers-Joseph-Shaffman condition, or a slip boundary condition (due to BeaversJoseph) on the interface. The tangential velocity is not continuous on the interface between them. The existence of solutions makes recourse to a fixed point procedure, but while the first boundary value problem is based on the abstract theory of quadratic problems under linear constraint the second one is based on a saddle-point approach. In isothermal and steady-state conditions, the well-posedness of a coupled StokesDarcy model with Beavers-Joseph-Shaffman and Beavers-Joseph interface boundary conditions are established, respectively, in [28] and, under the assumption of a small coefficient in the Beavers-Joseph interface boundary condition, in [8]. The study of interactions between flow and solidification within mushy layers can be found in [4].

In Section 2 we set the assumptions and the functional space framework, we define 
different problems and we state their existence results. In Sections 4, 5, and 7, we state some auxiliary results with a weak regularity hypothesis on either the coefficients or the domain. Sections 6 and 8 are devoted to the proofs of the presented results.

\section{Statement of the problem}

Let $\Omega_{f}$ and $\Omega_{p}$ be two disjoint bounded domains of $\mathbb{R}^{n}(n \geq 2)$ such that $\bar{\Omega}=$ $\bar{\Omega}_{f} \cup \bar{\Omega}_{p}$ is connected with Lipschitz boundary. The z-axis is taken as the perpendicular coordinate to a fixed ( $n-1)$-dimensional level and $\mathbf{e}_{z}$ is the unit vector in the direction of $z$. In geophysics, this represents the vertical direction. We use the notation $x \in \Omega$ such that $x=\left(x^{\prime}, z\right)$, with $x^{\prime}=\left(x_{1}, \cdots, x_{n-1}\right)$ and $z=x_{n}$.

Let $\Gamma=\partial \Omega_{f} \cap \Omega \subset \partial \Omega_{p}$ denote a $(n-1)$-dimensional interface that separates the fluid region from the porous medium. Define relatively open $(n-1)$-dimensional submanifolds $\Gamma_{i}=\partial \Omega_{i} \backslash \Gamma$, with meas $\left(\Gamma_{i}\right)>0,(i=f, p)$, where we will impose Dirichlet and Neumann boundary conditions. The flow velocity is in fact two velocities, namely $\mathbf{u}_{S}$ - the Stokes velocity calculated inside the fluid region, and $\mathbf{u}_{D}$ - the Darcy velocity calculated inside the porous medium. Here the subscripts $S$ and $D$ emphasize that these variables are for the Stokes and Darcy equations, respectively. We may omit these subscripts where the context is clear. While in the fluid part $\mathbf{u}$ and $\theta$ denote the usual velocity and temperature, in the porous media $\mathbf{u}$ is the superficial average quantity, and $\theta$ is the spatially averaged (over a representative elementary volume) microscopic quantity [35]. Precisely,

$$
\mathbf{u}_{D}=\frac{1}{\mathcal{V}} \int_{V_{f}} \mathbf{u}_{f} d x
$$

where $V_{f}$ is the fluid volume contained within an averaging volume $\mathcal{V}$ of the porous domain $\Omega_{p}$. Here it is understood that $V_{f}$ depends on position. The Darcy velocity is related to the usual (i.e. volume averaged over a volume element consisting of fluid only in the pores) velocity vector, that is, it is defined in terms of the intrinsic average velocity

$$
\mathbf{u}_{p}=\frac{1}{V_{f}} \int_{V_{f}} \mathbf{u}_{f} d x
$$

known as the pore velocity of the fluid, by $\mathbf{u}_{D}=\phi \mathbf{u}_{p}$, with $\phi$ denoting the porosity (i.e. the volume of fluid / total volume of porous body).

In porous media, due to the structure of the solid material in which the fluid lies, there can be a pronounced anisotropy in such parameters as permeability or thermal conductivity [30, 37.

2.1. Free (or natural convection) flow region. We begin by introducing the non-incompressibility condition which addresses viscous flows that can only undergo isochoric motions in isothermal processes, but can sustain motions that are not necessarily isochoric in processes that are not isothermal [25, 31]:

$$
\nabla \cdot \mathbf{u}=\alpha(\theta) \mathbf{u} \cdot \nabla \theta \quad \text { in } \Omega_{f} .
$$

Indeed, (2.1) comes from

$$
\operatorname{det} \mathbb{F}=f(\theta) \Leftrightarrow \nabla \cdot \mathbf{u}=\alpha(\theta) \frac{d \theta}{d t},
$$

where $\mathbb{F}$ is the deformation gradient, $f$ is a given function on the temperature $\theta, \mathbf{u}$ denotes the fluid velocity vector, $\alpha=f^{\prime}(\theta) / f(\theta)$ represents the coefficient of thermal 
expansion, the prime denotes the derivative with respect to the argument, and the steady-state material derivative is $d / d t=\mathbf{u} \cdot \nabla$ when the Eulerian description is taken care of. The particular case $\alpha(\theta)=1 / \theta$ in (2.1), or equivalently $f \equiv$ id, corresponds to the weakly compressible flow model [32, p. 278].

The assumption that we are in the presence of slow motion legitimates the use of the Stokes model, which assumes that the inertial forces are negligible compared with viscous forces and then the convective term to be neglected. Notice that the Stokes system also models flows with high viscosity or at small length scales. The free fluid is governed by the Stokes equations, with the Oberbeck-Boussinesq approximation (for steady processes)

$$
-\nabla \cdot \tau=-\nabla p+\mathbf{f}(\cdot, \theta) \quad \text { in } \Omega_{f} .
$$

Here and in the sequel, the dot indicates the dependence of the function on the spatial variable and it will be omitted whenever there can be no ambiguity. In the motion equation (2.2), $p$ represents the pressure, $\tau=p I+\sigma$ denotes the deviatoric stress tensor, where $\sigma$ represents the Cauchy stress tensor and $I$ is the identity matrix, and $\mathbf{f}$ represents the buoyancy force, i.e.

$$
\mathbf{f}=-\left(\theta-\theta_{r}\right) \rho \alpha g \mathbf{e}_{z},
$$

with $-g \mathbf{e}_{z}$ representing the gravitational force, $\rho$ denoting the density, and $\theta_{r}$ standing for a reference temperature.

Here we consider the Newtonian fluid (linearly viscous fluid), that is, the constitutive law for $\tau$ is

$$
\tau=\mu(\theta) D \mathbf{u}+\lambda(\theta) \operatorname{tr}(D \mathbf{u}) I, \quad \operatorname{tr}(D \mathbf{u})=I: D \mathbf{u}=\nabla \cdot \mathbf{u},
$$

where $\mu$ and $\lambda$ are the viscosity coefficients (dynamic and bulk viscosities, respectively) in accordance with the second law of thermodynamics

$$
\mu(\theta) \geq \mu_{\#}>0, \quad n \lambda(\theta)+\mu(\theta) \geq 0
$$

and the symmetrized gradient $D=\left(\nabla+\nabla^{T}\right) / 2$. Here we denote $\zeta: \varsigma=\zeta_{i j} \varsigma_{i j}$, taking into account the convention on implicit summation over repeated indices.

2.2. Saturated porous medium. For non-isothermal flow, the Darcy equation consists of

$$
\begin{aligned}
\mathbf{u}_{D} & =-\frac{\Pi}{\mu}\left(\nabla p-\rho g \mathbf{e}_{z}\right) \\
\nabla \cdot \mathbf{u}_{D} & =0 \quad \text { in } \Omega_{p},
\end{aligned}
$$

corresponding to the Oberbeck-Boussinesq approximation [23]. Here $\Pi$ represents the permeability, $p$ is the intrinsic average pressure, and $\rho$ the density of the fluid in $\Omega_{p}$. The permeability tensor of the porous media, which is assumed to be symmetric and positive definite, may be location dependent (heterogeneous). Indeed, it depends on space and temperature through the porosity $\phi$. For instance, the Carman-Kozeny permeability function [4, 9] $\Pi=\Pi_{0} \phi^{3} /(1-\phi)^{2}$, where $\Pi_{0}$ is a reference permeability. In geological situations, the permeability usually decreases with depth and the porosity is usually a function of the $\operatorname{depth}(z)$ (e.g. see [36]). For simplicity, set $\mathbf{g}(\theta)=-\rho(\theta) g \mathbf{e}_{z}$. 
Groundwater systems, such as aquifers, are mostly made up of a matrix, i.e., a porous medium, that holds the water. The Darcy velocity field is proportional to the gradient of the piezometric head $h$. The latter is defined as $h=z+p /(\rho g)$ where $z$ is the vertical distance defined from a reference level and $g$ indicates the gravity acceleration constant. The Darcy law states that

$$
\mathbf{u}_{D}=-\mathbf{K} \nabla h,
$$

where $\mathbf{K}$ is the hydraulic conductivity tensor and obeys the relation $\mathbf{K}=\Pi \rho g / \mu$. In the case of isothermal porous media, $\rho$ is constant and (2.5) and (2.7) coincide. Otherwise, the Darcy law (2.7) reads

$$
\mathbf{u}_{D}=-\frac{\Pi \rho g}{\mu}\left(\mathbf{e}_{z}+\frac{\nabla p}{\rho g}-\frac{p}{\rho^{2} g} \nabla \rho\right)=-\frac{\Pi}{\mu}\left(\rho g \mathbf{e}_{z}+\nabla p-p \frac{\nabla \rho}{\rho}\right) .
$$

2.3. Heat transfer model. In order to deduce an accurate formulation of the problem under the compressible condition (2.1), let us recall that the specific (i.e. per unit mass) internal energy $e$ is, via the Maxwell equation, given by

$$
e(\theta, \rho)=\int_{\theta_{r}}^{\theta} c_{v}(z) d z+\int_{\rho_{r}}^{\rho} \frac{P_{E}(z)}{z^{2}} d z
$$

where $c_{v}$ denotes the specific heat capacity of the fluid at constant volume

$$
c_{v}(\theta)=\frac{\partial e}{\partial \theta}(\theta, \rho)>0,
$$

and $P_{E}$ is the elastic part related to the action of intermolecular forces. Then, applying the continuity equation, the energy equation reads

$$
\begin{aligned}
\nabla \cdot(\mathbb{K} \nabla \theta) & =\rho \mathbf{u} \cdot\left(c_{v}(\theta) \nabla \theta+\frac{P_{E}(\rho)}{\rho^{2}} \nabla \rho\right) \\
& =\rho c_{v}(\theta) \mathbf{u} \cdot \nabla \theta-P_{E}(\rho) \nabla \cdot \mathbf{u},
\end{aligned}
$$

with $\mathbb{K}$ denoting the thermal conductivity tensor. Notice that $\mathbb{K}$ is a function depending only on the temperature at each subdomain, that is, the Fourier law inducing the heat flux is $\mathbf{q}_{i}=-\mathbb{K}_{i} \nabla \theta_{i}$ in $\Omega_{i}(i=f, p)$. Precisely, (2.1) and (2.6) imply that (2.8) reads

$$
\varkappa(\theta) \mathbf{u} \cdot \nabla \theta-\nabla \cdot(\mathbb{K}(\theta) \nabla \theta)=0,
$$

with $\varkappa(\theta)=\rho(\theta) c_{v}(\theta)-P_{E}(\rho(\theta)) \alpha(\theta)$ in $\Omega_{f}$ and $\varkappa(\theta)=\rho(\theta) c_{v}(\theta)$ in $\Omega_{p}$.

On the other hand, using (2.1), the density is given by

$$
\rho(x, t)=\rho_{0}(x) \exp \left[-\ln (f(\theta(x, t)))+\ln \left(f\left(\theta_{0}(x)\right)\right)\right]=\frac{\rho_{0}(x) f\left(\theta_{0}(x)\right)}{f(\theta(x, t))}>0,
$$

applying the method of characteristics. At steady-state, we obtain the explicit formula

$$
\rho(x, e)=\frac{\rho_{0}(x) f\left(\theta_{0}(x)\right)}{f(e)}, \quad \text { a.e. } x \in \Omega, \forall e \in \mathbb{R} .
$$

Therefore, it satisfies

$$
\frac{\partial \rho}{\partial e}(\cdot, e)=-\alpha(e) \rho .
$$

Simplified assumptions on the density (i.e. that it is linear or quadratic in temperature) are used in [23] and [30], respectively. 
2.4. Outer boundaries. We denote by $\Gamma_{f}$ and $\Gamma_{p}$ the outer boundaries of $\Omega_{f}$ and $\Omega_{p}$, respectively, which satisfy

$$
\Gamma_{f} \cap \Gamma_{p}=\emptyset, \quad \bar{\Gamma}_{f} \cup \bar{\Gamma}_{p}=\partial \Omega, \quad \Sigma=\bar{\Gamma}_{f} \cap \bar{\Gamma}_{p},
$$

with $\Sigma$ representing a $(n-2)$-dimensional manifold. Both boundaries exchange heat with the environment. Here, we restrict ourselves to two situations of the temperature obeying the homogeneous boundary condition, depending to a parameter $\gamma=0,1$,

$$
\begin{aligned}
\gamma(\mathbb{K}(\theta) \nabla \theta) \cdot \mathbf{n}_{f}+(1-\gamma) \theta & =0 & & \text { on } \Gamma_{f} ; \\
\nabla \theta \cdot \mathbf{n}_{p} & =0 & & \text { on } \Gamma_{p},
\end{aligned}
$$

where $\mathbf{n}_{i}$ stands for the unit outward vector to $\Gamma_{i}(i=f, p)$. The condition (2.10) corresponds to the Dirichlet and the Neumann boundary conditions as well as $\gamma=0$ and $\gamma=1$, respectively. Consequently, (2.10)-(2.11) describe the Zaremba and the Neumann situations.

On the boundary confining the fluid we assume the following Dirichlet condition:

$$
\mathbf{u}=\mathbf{u}_{0} \quad \text { on } \Gamma_{f} .
$$

In this work we consider the homogeneous prescribed situation, that is, $\mathbf{u}_{0}=\mathbf{0}$, since the general case can be handled by subtracting the background profile that fits the specified velocity at the rigid surface.

On the outer boundary of the porous medium, we assume

$$
\mathbf{u}_{D} \cdot \mathbf{n}_{p}=0 \quad \text { on } \Gamma_{p},
$$

which gives, by (2.7), the homogeneous Neumann boundary condition

$$
(\mathbf{K}(\theta) \nabla h) \cdot \mathbf{n}_{p}=0 \quad \text { on } \Gamma_{p} .
$$

2.5. Interface boundary. The interface conditions are, respectively, the continuity of mass flux and the continuity of normal stresses,

$$
\begin{aligned}
\mathbf{u}_{S} \cdot \mathbf{n} & =\mathbf{u}_{D} \cdot \mathbf{n} ; \\
\tau_{N} & =[p],
\end{aligned}
$$

where $\mathbf{n}$ denotes the unit normal to $\Gamma$ pointing from the fluid region to the porous medium and [.] denotes the jump of a quantity across the interface in direction of $-\mathbf{n}$, i.e. $[v]:=v_{f}-v_{p}$. Recall that $u_{N}$ and $\mathbf{u}_{T}$ are the normal and the tangential components of the velocity vector, respectively, and $\tau_{N}=(\tau \cdot \mathbf{n}) \cdot \mathbf{n}$ and $\tau_{T}=\tau \cdot \mathbf{n}-\tau_{N} \mathbf{n}$ are the normal and the tangential components of the deviatoric stress tensor $\tau$.

The first condition guarantees the conservation of mass, i.e., the exchange of fluid between the two domains is conservative. The second condition represents the balance of the two normal Cauchy stress vectors $\sigma_{f N}+\sigma_{p N}=0$.

The boundary conditions for constant interface temperature are clear; both regions should have a temperature that equals a prescribed interface temperature, or simply

$$
\theta_{f}=\theta_{p} \quad \text { on } \Gamma .
$$

The heat transfer transmission is also constituted by the continuous heat flux condition

$$
\left(\mathbb{K}_{f}\left(\theta_{f}\right) \nabla \theta_{f}\right) \cdot \mathbf{n}=\left(\mathbb{K}_{p}\left(\theta_{p}\right) \nabla \theta_{p}\right) \cdot \mathbf{n} \quad \text { on } \Gamma \text {. }
$$


Finally, we assume the existence of the jump between the Stokes and Darcy velocities:

$$
\tau_{T}=-\beta\left[\mathbf{u}_{T}\right],
$$

usually written in the following form, which is known as the Beavers-Joseph interface boundary condition or the Beavers-Joseph condition on the tangential stress [5]:

$$
(\tau \cdot \mathbf{n}) \cdot \mathbf{t}_{i}=-\beta\left(\mathbf{u}_{S}-\mathbf{u}_{D}\right) \cdot \mathbf{t}_{i}
$$

where the coefficient $\beta=\alpha_{B J}\left(\mathbf{t}_{i} \cdot \Pi \mathbf{t}_{i}\right)^{-1 / 2}>0$ denotes the Beavers-Joseph slip coefficient, where $\alpha_{B J}$ is dimensionless and characterizes the nature of the porous surface, $\Pi$ the anisotropic permeability and $\left\{\mathbf{t}_{i}\right\}_{i=1, \cdots, n-1}$ represents an orthonormal system of tangential vector spaces to the interface.

Whenever the filtration velocity is usually much smaller than the slip velocity [16], the simplified version of the Beavers-Joseph condition that contains only variables in the free fluid domain $\Omega_{f}$,

$$
\tau_{T}=-\beta \mathbf{u}_{T},
$$

is the so-called Beavers-Joseph-Saffman condition [27.

In conclusion, we call by Beavers-Joseph-Saffman-Stokes-Darcy-Fourier (BJSSDF) problem the Stokes-Darcy-Fourier system (2.1)-(2.3), (2.5)-(2.6), (2.9)-(2.13), (2.15)-(2.18) under the Beavers-Joseph-Saffman interface boundary condition (2.20). The Stokes-Darcy-Fourier system (2.1)-(2.3), (2.5)-(2.7), (2.9)-(2.12), (2.14)-(2.18) under the Beavers-Joseph interface boundary condition (2.19) we call the BeaversJoseph-Stokes-Darcy-Fourier (BJ-SDF) problem.

\section{Existence results}

Recall that $\Omega$ is a Lipschitz domain if it is a bounded connected open set of $\mathbb{R}^{n}$ with its boundary locally described by means of (appropriately rotated and translated) graphs of real-valued Lipschitz functions. We assume that $\mu, \lambda, \rho, \alpha, \varkappa: \Omega \times \mathbb{R} \rightarrow \mathbb{R}$ are Carathéodory functions, that is, measurable with respect to $x \in \Omega$ for every $e \in \mathbb{R}$, continuous with respect to $e \in \mathbb{R}$ almost every $x \in \Omega$ such that (2.4) is satisfied, and

$$
\begin{array}{ll}
\exists \mu^{\#}>0: & \mu(\cdot, e) \leq \mu^{\#}, \quad \forall e \in \mathbb{R}, \text { a.e. in } \Omega ; \\
\exists \lambda^{\#}>0: & \lambda(\cdot, e) \leq \lambda^{\#}, \quad \forall e \in \mathbb{R}, \text { a.e. in } \Omega ; \\
\exists \rho^{\#}>0: & |\rho(\cdot, e)| \leq \rho^{\#}, \quad \forall e \in \mathbb{R}, \text { a.e. in } \Omega ; \\
\exists \alpha^{\#}>0: & |\alpha(\cdot, e)| \leq \alpha^{\#}, \quad \forall e \in \mathbb{R}, \text { a.e. in } \Omega ; \\
\exists \varkappa^{\#}>0: & |\varkappa(\cdot, e)| \leq \varkappa^{\#}, \quad \forall e \in \mathbb{R}, \text { a.e. in } \Omega,
\end{array}
$$

and $\beta: \Gamma \times \mathbb{R} \rightarrow \mathbb{R}$ is a Carathéodory function such that

$$
\exists \beta^{\#}>0: \quad|\beta(\cdot, e)| \leq \beta^{\#}, \quad \forall e \in \mathbb{R}, \text { a.e. on } \Gamma .
$$

Finally we see that the functions $\mathbf{f}, \mathbf{g} \in \mathbf{C}(\Omega \times \mathbb{R})$ are such that

$$
\begin{aligned}
& |\mathbf{f}(\cdot, e)| \leq f^{\#}(|e|+1) ; \\
& |\mathbf{g}(\cdot, e)| \leq \rho^{\#} g
\end{aligned}
$$

with $f^{\#}=g \rho^{\#} \alpha^{\#} \max \left\{\theta_{r}, 1\right\}$. 
Let us define the Banach spaces, for $r>1$ and $\gamma=0,1$,

$$
\begin{aligned}
& W_{\gamma, \Gamma_{f}}^{1, r}(\Omega)=\left\{v \in W^{1, r}(\Omega): v_{f}:=\left.v\right|_{\Omega_{f}}, v_{p}:=\left.v\right|_{\Omega_{p}}, v_{f}=v_{p} \text { on } \Gamma,(1-\gamma) v_{f}=0 \text { on } \Gamma_{f}\right\} ; \\
& \mathbf{H}_{\Gamma_{f}}^{1}\left(\Omega_{f}\right)=\left\{\mathbf{v} \in \mathbf{H}^{1}\left(\Omega_{f}\right):\left.\mathbf{v}\right|_{\Gamma_{f}}=0\right\} ; \\
& \mathbf{H}\left(\operatorname{div}, \Omega_{p}\right)=\left\{\mathbf{v} \in \mathbf{L}^{2}\left(\Omega_{p}\right): \nabla \cdot \mathbf{v} \in L^{2}\left(\Omega_{p}\right)\right\} ; \\
& \mathbf{H}=\left\{\mathbf{v} \in \mathbf{H}\left(\operatorname{div}, \Omega_{p}\right): \nabla \cdot \mathbf{v}=0 \text { in } \Omega_{p}, \mathbf{v} \cdot \mathbf{n}_{p}=0 \text { on } \Gamma_{p}\right\} ; \\
& \mathbf{V}=\left\{\mathbf{v}=\left(\mathbf{v}_{f}, \mathbf{v}_{p}\right) \in \mathbf{H}_{\Gamma_{f}}^{1}\left(\Omega_{f}\right) \times \mathbf{H}\left(\operatorname{div}, \Omega_{p}\right): \mathbf{v}_{p} \cdot \mathbf{n}_{p}=0 \text { on } \Gamma_{p},\right. \\
&\left.\quad\left(\mathbf{v}_{f}-\mathbf{v}_{p}\right) \cdot \mathbf{n}=0 \text { on } \Gamma\right\} ; \\
& Q=\{q=\left.\left(q_{f}, q_{p}\right) \in L^{2}\left(\Omega_{f}\right) \times L^{2}\left(\Omega_{p}\right): \int_{\Omega_{f}} q_{f} d x+\int_{\Omega_{p}} q_{p} d x=0\right\} .
\end{aligned}
$$

Considering that the Poincaré inequality occurs when $\Gamma_{f} \neq \emptyset$, then the above Hilbert spaces are endowed with the standard seminorms. In particular, we get

$$
\|\mathbf{v}\|_{\mathbf{v}}=\left\|D \mathbf{v}_{f}\right\|_{2, \Omega_{f}}+\left\|\mathbf{v}_{p}\right\|_{2, \Omega_{p}}+\left\|\nabla \cdot \mathbf{v}_{p}\right\|_{2, \Omega_{p}}
$$

For a Lipschitz domain $\Omega_{f}$, the trace operator $H_{\Gamma_{f}}^{1}\left(\Omega_{f}\right) \rightarrow H_{00}^{1 / 2}(\Gamma)$ has bounded linear right inverse, that is, for every element $v$ of the trace space

$$
H_{00}^{1 / 2}(\Gamma)=\left\{v \in L^{2}(\Gamma) \text { : its zero extension belongs to } H^{1 / 2}\left(\partial \Omega_{f}\right)\right\}
$$

there exists $u_{f} \in H_{\Gamma_{f}}^{1}\left(\Omega_{f}\right)$ such that $u_{f}=v$ on $\Gamma$ [20]. However, the trace operator, considered as a mapping from $H_{\Gamma_{f}}^{1}\left(\Omega_{f}\right)$ in $L^{2}\left(\partial \Omega_{f}\right)$, does not belong to $H_{0}^{1 / 2}(\Gamma)$. Recall that an element of $H^{1 / 2}(\Gamma)$ cannot be extended by zero outside $\Gamma$ to a function in $H^{1 / 2}(\partial \Omega)$. Notice that $H_{00}^{1 / 2}(\Gamma)$ is isomorphic to the orthogonal complement of $H_{0}^{1}\left(\Omega_{f}\right)$ in $H_{\Gamma_{f}}^{1}\left(\Omega_{f}\right)$. Thus,

$$
H_{00}^{1 / 2}(\Gamma) \sim\left\{v \in H^{1 / 2}\left(\partial \Omega_{f}\right): v=0 \quad \text { on } \Gamma_{f}\right\} .
$$

The meaning of the conditions $\mathbf{v} \cdot \mathbf{n}_{p}=0$ on $\Gamma_{p}$ and $\mathbf{v} \cdot \mathbf{n}=0$ on $\Gamma$ should be understood, respectively, as

$$
\begin{array}{r}
\left\langle\mathbf{v} \cdot \mathbf{n}_{p}, v\right\rangle_{\Gamma_{p}}=0 \quad \forall v \in H_{00}^{1 / 2}\left(\Gamma_{p}\right) ; \\
\langle\mathbf{v} \cdot \mathbf{n}, v\rangle_{\Gamma}=0, \quad \forall v \in H_{00}^{1 / 2}(\Gamma),
\end{array}
$$

where the symbols $\langle\cdot, \cdot\rangle_{\Gamma_{p}}$ and $\langle\cdot, \cdot\rangle_{\Gamma}$ stand for the duality pairing $\langle\cdot, \cdot\rangle_{Y^{\prime} \times Y}$, using the notations $Y=H_{00}^{1 / 2}\left(\Gamma_{p}\right)$ and $Y=H_{00}^{1 / 2}(\Gamma)$, respectively.

REMARK 3.1. If $\Omega$ is of class $C^{0,1}$, then the following embeddings hold:

$$
\begin{aligned}
W^{m, p}(\Omega) \hookrightarrow C^{k, m-k-n / p}(\bar{\Omega}) & \text { if } k<m-n / p<k+1 ; \\
W^{m, p}(\Omega) \hookrightarrow W^{s, p n /(n-(m-s) p)}(\Omega) & \text { if } m \geq s \text { and }(m-s) p<n ; \\
W^{1-1 / p, p}(\partial \Omega) \hookrightarrow L^{p(n-1) /(n-p)}(\partial \Omega) & \text { if } p<n .
\end{aligned}
$$

The vector field $\mathbf{n}$ is of class $C^{m-1,1}$ for bounded domains of class $C^{m, 1}$. Indeed, the unit outward normal vector to the boundary of $C^{0,1}$ domains belongs only to $\mathbf{L}^{\infty}(\partial \Omega)$.

In the sequel, some problems are defined and their existence results are stated. 
Definition 3.2 (Beavers-Joseph-Saffman-Stokes-Darcy-Fourier problem). We say that $\left(\mathbf{u}_{S}, \mathbf{u}_{D}, p, \theta\right) \in \mathbf{V} \times Q \times W_{\gamma, \Gamma_{f}}^{1, r}(\Omega)$, for some $r>n$, is a weak solution to the $B J S-S D F$ problem if it satisfies

$$
\begin{aligned}
& \int_{\Omega_{f}} \mu(\theta) D \mathbf{u}_{S}: D \mathbf{v}_{f} d x+\int_{\Omega_{f}} \lambda(\theta) \nabla \cdot \mathbf{u}_{S} \nabla \cdot \mathbf{v}_{f} d x \\
& \quad+\int_{\Omega_{p}} \mu(\theta) \Pi^{-1}(\theta) \mathbf{u}_{D} \cdot \mathbf{v}_{p} d x+\int_{\Gamma} \beta(\theta) \mathbf{u}_{S} \cdot \mathbf{v}_{f} d s \\
& =\int_{\Omega} p \nabla \cdot \mathbf{v} d x+\int_{\Omega_{f}} \mathbf{f}(\theta) \cdot \mathbf{v}_{f} d x+\int_{\Omega_{p}} \mathbf{g}(\theta) \cdot \mathbf{v}_{p} d x, \quad \forall \mathbf{v} \in \mathbf{V} ; \\
& \int_{\Omega} \varkappa(\theta) \mathbf{u} \cdot \nabla \theta \psi d x+\int_{\Omega}(\mathbb{K}(\theta) \nabla \theta) \cdot \nabla \psi d x=0, \quad \forall \psi \in W_{\gamma, \Gamma_{f}}^{1, r^{\prime}}(\Omega) ; \\
& \int_{\Omega} \nabla \cdot \mathbf{u} q d x=\int_{\Omega_{f}} \alpha(\theta) \mathbf{u}_{S} \cdot \nabla \theta q d x, \quad \forall q \in Q,
\end{aligned}
$$

with $r^{\prime}=r /(r-1)$ being the conjugate exponent of $r$.

The following definitions for the domain are essential to provide the regularity of the solutions of auxiliary boundary value problems.

Definition 3.3 ([21]). We say that any set $\Omega \subset \mathbb{R}^{n}$ is regular if $\Omega$ is bounded and if for every $y \in \partial \Omega$ there exist open subsets $U$ and $\widetilde{U}$ of $\mathbb{R}^{n}$ and a bijection $a: U \rightarrow \widetilde{U}$ such that $a$ and $a^{-1}$ are Lipschitzian, $y \in U$, and $a(U \cap \Omega)$ is one of the following sets:

$$
\begin{aligned}
& E_{1}=\left\{x \in \mathbb{R}^{n}:|x|<1, x_{n}<0\right\} \\
& E_{2}=\left\{x \in \mathbb{R}^{n}:|x|<1, x_{n} \leq 0\right\} ; \\
& E_{3}=\left\{x \in E_{2}:|x|<1, x_{n} \leq 0 \text { or } x_{1}>0\right\} .
\end{aligned}
$$

DeFINITION $3.4([14,15])$. We say that any set $\Omega \subset \mathbb{R}^{n}$ is Lipschitz with constant $\delta$ if there is a constant $\left.\left.R_{0} \in\right] 0,1\right]$ such that, for any $y \in \partial \Omega$ and $\left.\left.r \in\right] 0, R_{0}\right]$, there exists a Lipschitz function $a: \mathbb{R}^{n-1} \rightarrow \mathbb{R}$ satisfying

$$
\Omega \cap B(y, r)=\left\{x=\left(x^{\prime}, x_{n}\right) \in B(y, r): x_{n}>a\left(x^{\prime}\right)\right\}
$$

and

$$
\sup \left\{\frac{\left|a\left(x^{\prime}\right)-a\left(z^{\prime}\right)\right|}{\left|x^{\prime}-z^{\prime}\right|}: x^{\prime}, z^{\prime} \in B\left(y^{\prime}, R_{0}\right) x^{\prime} \neq z^{\prime}\right\} \leq \delta
$$

in some coordinate system.

Note that all $C^{1}$ domains satisfy the above assumption on $\Omega$ for any $\delta>0$.

Definition 3.5 ([7]). We say that any set $\Omega \subset \mathbb{R}^{n}$ is $\delta$-Reifenberg flat if there is a constant $R_{0}>0$ such that, for every $y \in \partial \Omega$ and every $\left.\left.r \in\right] 0, R_{0}\right]$, there exists a coordinate system such that $y=0$ in this coordinate system and that

$$
\left\{x \in B(0, r): x_{n}>\delta r\right\} \subset B(0, r) \cap \Omega \subset\left\{x \in B(0, r): x_{n}>-\delta r\right\} .
$$

We state the first existence result provided that $\Pi^{-1}$ and $\mathbb{K}$ are assumed to be symmetric positive definite matrices. 
THEOREM 3.6. Suppose that all above assumptions, namely (3.1)-(3.8), are fulfilled. Let $n=2$ and $\Omega$ be regular (cf. Definition 3.3). If in addition $\Pi^{-1}: \Omega_{p} \times \mathbb{R} \rightarrow \mathbb{M}_{\mathrm{sym}}^{n \times n}$ and $\mathbb{K}: \Omega \times \mathbb{R} \rightarrow \mathbb{M}_{\text {sym }}^{n \times n}$ are Carathéodory functions such that

$$
\begin{array}{ll}
\exists \pi^{\#}>0: & \sum_{i, j=1}^{n}\left|\left(\Pi^{-1}\right)_{i j}(\cdot, e)\right| \leq \pi^{\#} \quad \forall e \in \mathbb{R}, \text { a.e. in } \Omega_{p} ; \\
\exists \pi_{\#}>0: & \left(\Pi^{-1}\right)_{i j}(\cdot, e) b_{i} b_{j} \geq \pi_{\#}|\mathbf{b}|^{2} \quad \forall \mathbf{b} \in \mathbb{R}^{n}, e \in \mathbb{R}, \text { a.e. in } \Omega_{p} ; \\
\exists k^{\#}>0: & \sum_{i, j=1}^{n}\left|K_{i j}(\cdot, e)\right| \leq k^{\#} \quad \forall e \in \mathbb{R}, \text { a.e. in } \Omega ; \\
\exists k_{\#}>0: & K_{i j}(\cdot, e) b_{i} b_{j} \geq k_{\#}|\mathbf{b}|^{2} \quad \forall \mathbf{b} \in \mathbb{R}^{n}, e \in \mathbb{R}, \text { a.e. in } \Omega,
\end{array}
$$

where $\mathbb{M}_{\mathrm{sym}}^{n \times n}$ is the set of symmetric matrices of the type $n \times n$, then there exists at least one weak solution $\left(\mathbf{u}_{S}, \mathbf{u}_{D}, p, \theta\right) \in \mathbf{V} \times Q \times W_{\gamma, \Gamma_{f}}^{1,2+\varepsilon}(\Omega)$ in accordance with Definition 3.2. for some $\varepsilon=\varepsilon(\partial \Omega)>0$.

When $\Sigma \neq \emptyset$, the solution of the mixed Dirichlet-Neumann boundary problem for the Laplace equation may be singular even if it has a very regular right-hand side. A geometrical configuration with an inner angle between the Dirichlet and Neumann boundaries measuring $\pi$ generates a singularity which behaves locally like the function $\operatorname{Im} \sqrt{z}$. This function is harmonic in an appropriate bounded domain $\Omega \subset \mathbb{R} \times \mathbb{R}^{+}$, satisfies the homogeneous Dirichlet condition on $\mathbb{R}_{0}^{+} \times\{0\}$ and the homogeneous Neumann condition on $\mathbb{R}^{-} \times\{0\}$, is not locally $H^{2}$ in any neighborhood of the origin, and its normal derivative is not locally $L^{2}$ along the boundary. Thus the mixed problem $(\gamma=0)$ has recourse to the optimal regularity of the elliptic problem where mixed boundary conditions and piecewise constant coefficient occur simultaneously. We refer to 22 for the study of prototypical situations (such as $\Omega$ is a bounded domain and its closure $\bar{\Omega}$ is a polyhedral 3-manifold with the same boundary of $\Omega$ ) of the operator

$$
\nabla \cdot(\mathbb{A} \nabla): W_{0, \Gamma_{f}}^{1, r}(\Omega) \longrightarrow\left(W_{0, \Gamma_{f}}^{1, r}(\Omega)\right)^{\prime}
$$

which is a topological isomorphism for some $r>3$ whenever the coefficient function $\mathbb{A}$ is elliptic and takes its piecewise constant values in the set of real, symmetric, positive definite $3 \times 3$ matrices. Indeed, the coefficient function is constant on the interior of each 3-cell belonging to a finite triangulation of $\bar{\Omega}$. Our 3D result may be also extended to this particular situation. However, for a clear presentation, we restrict ourselves to the Neumann situation.

For $n=3$, let us state the particular Neumann problem $(\gamma=1)$. For a real function $f$, we let $(f)_{B}$ stand for the integral average of $f$ over the ball $B$ (which will be explicitly considered).

THEOREm 3.7. Suppose that the assumptions (3.1)-(3.8) and (3.12)-(3.15) are fulfilled. Let $n=3$. Then there exist small positive constants $\delta$ and $\iota$ such that

1. $\Omega$ satisfies Definition 3.5, and for the same constant $R_{0}>0$ the components $A_{i j}$ of the Nemytskii operator $\mathbb{A}=\mathbb{K}(e) \in \mathbf{L}^{\infty}(\Omega)$ satisfy

$$
\sup _{x \in \mathbb{R}^{3}} \sup _{0<r \leq R_{0}} \frac{1}{|B(x, r)|} \int_{B(x, r)}\left|A_{i j}(y)-\left(A_{i j}\right)_{B(x, r)}\right|^{2} d y \leq \iota^{2} ;
$$

2. $\Omega$ satisfies Definition 3.4, and the Nemytskii operator $\mathbb{A}=\mathbb{K}(e) \in \mathbf{L}^{\infty}(\Omega)$ satisfies 
(a) There is a constant $\left.\left.R_{1} \in\right] 0,1\right]$ such that, for any $x \in \mathbb{R}^{3}$ and any $\left.\left.r \in\right] 0, R_{1}\right]$,

$$
\sup _{i j} \frac{1}{2 r} \int_{x_{n}-r}^{x_{n}+r} \frac{1}{\left|B\left(x^{\prime}, r\right)\right|} \int_{B\left(x^{\prime}, r\right)}\left|A_{i j}(y)-\left(A_{i j}\right)_{B\left(x^{\prime}, r\right)}\left(y_{n}\right)\right| d y^{\prime} d y_{n} \leq \iota ;
$$

(b) There is a constant $\left.\left.R_{2} \in\right] 0,1\right]$ such that, for any $x \in \mathbb{R}^{3}$ with $\operatorname{dist}(x, \partial \Omega) \leq$ $R_{2}$ and any $\left.\left.r \in\right] 0, R_{2}\right]$,

$$
\sup _{i j} \frac{1}{|B(x, r)|} \int_{B(x, r)}\left|A_{i j}(y)-\left(A_{i j}\right)_{B(x, r)}\right| d y \leq \iota,
$$

and there is at least one weak solution $\left(\mathbf{u}_{S}, \mathbf{u}_{D}, p, \theta\right) \in \mathbf{V} \times Q \times W^{1,4}(\Omega)$ in accordance with Definition 3.2.

The results of Theorem 3.6 and 3.7 may lead to the counter-intuitive claim that the regularity increases as the dimension increases. It seems that the regularity of $\theta$ is worse in 2D $\left(W^{1,2+\varepsilon}(0<\varepsilon<1)\right.$, under regular domains in accordance with Definition 3.3) than in 3D ( $W^{1,4}$, under domains in accordance with Definition 3.5 or Definition 3.4). However, notice that the $2 \mathrm{D}$ domain is less smooth than the $3 \mathrm{D}$ domain. In this work, we focus our attention on the weaker assumptions on the smoothness of the domain - which are of fundamental significance in many application areas - and as a consequence the optimal regularity of the temperature in such domains.

REMARK 3.8. The conditions 1 and 2 in Theorem 3.7 by no means imply that the coefficient function must belong the John-Nirenberg space BMO of the functions of bounded mean oscillation with locally small BMO semi-norm. Indeed, in (a) the leading coefficient is assumed to be measurable in one direction and has small BMO semi-norm in the other directions, and in (b) it has small BMO semi-norm in a neighborhood of the boundary of the domain.

Next, let us introduce the second problem under study.

Definition 3.9 (Beavers-Joseph-Stokes-Darcy-Fourier problem). We say that $(\mathbf{u}, p, h, \theta) \in \mathbf{H}_{\Gamma_{f}}^{1}\left(\Omega_{f}\right) \times L^{2}\left(\Omega_{f}\right) \times H_{\Gamma_{p}}^{1}\left(\Omega_{p}\right) \times W_{\gamma, \Gamma_{f}}^{1, r}(\Omega)$, for some $r>n$, is a weak solution to the BJ-SDF problem if it satisfies

$$
\begin{aligned}
& \int_{\Omega_{f}} \mu(\theta) D \mathbf{u}: D \mathbf{v} d x+\int_{\Omega_{f}} \lambda(\theta) \nabla \cdot \mathbf{u} \nabla \cdot \mathbf{v} d x+\int_{\Omega_{p}}(\mathbf{K}(\theta) \nabla h) \cdot \nabla v d x \\
& \quad+\int_{\Gamma} h \mathbf{v} \cdot \mathbf{n} d s-\int_{\Gamma} \mathbf{u} \cdot \mathbf{n} v d s+\int_{\Gamma} \beta(\theta) \mathbf{u} \cdot \mathbf{v} d s+\langle\beta(\theta) \mathbf{K}(\theta) \nabla h, \mathbf{v}\rangle_{\Gamma} \\
& =\int_{\Omega_{f}} p \nabla \cdot \mathbf{v} d x+\int_{\Omega_{f}} \mathbf{f}(\theta) \cdot \mathbf{v} d x, \quad \forall(\mathbf{v}, v) \in \mathbf{H}_{\Gamma_{f}}^{1}\left(\Omega_{f}\right) \times H_{\Gamma_{p}}^{1}\left(\Omega_{p}\right) ; \\
& \int_{\Omega}(\mathbb{K}(\theta) \nabla \theta) \cdot \nabla \psi d x+\int_{\Omega_{f}} \varkappa(\theta) \mathbf{u} \cdot \nabla \theta \psi d x \\
& \quad+\int_{\Omega_{p}} \varkappa(\theta)(\mathbf{K}(\theta) \nabla h) \cdot \nabla \theta \psi d x=0, \quad \forall \psi \in W_{\gamma, \Gamma_{f}}^{1, r^{\prime}}(\Omega) ; \\
& \int_{\Omega_{f}} \nabla \cdot \mathbf{u} q d x=\int_{\Omega_{f}} \alpha(\theta) \mathbf{u} \cdot \nabla \theta q d x, \quad \forall q \in L^{2}\left(\Omega_{f}\right) .
\end{aligned}
$$

Let us restrict the study of the BJ-SDF problem to the two-dimensional case where the thermal conductivity $\mathbb{K}$ is a symmetric positive definite matrix with Carathéodory 
components. The corresponding three-dimensional case should be treated under stronger assumptions on the character of the domain and the thermal conductivity coefficient (cf. Theorem 3.7).

Theorem 3.10. Let $n=2$ and $\Omega$ be regular (cf. Definition 3.3). Suppose that the assumptions (3.1)-(3.8) and (3.14)-(3.15) are fulfilled, $\beta \in C^{0,1}(\bar{\Gamma} \times \mathbb{R})$, and the leading coefficient $\mathbf{K} \in \mathbf{C}\left(\bar{\Omega}_{p} \times \mathbb{R}\right)$ is such that the following coercivity and Lipschitz continuity conditions are satisfied:

$$
\begin{array}{ll}
\exists \pi_{\#}>0: & K_{i j}(\cdot, e) b_{i} b_{j} \geq \pi_{\#}|\mathbf{b}|^{2} \quad \forall \mathbf{b} \in \mathbb{R}^{n}, e \in \mathbb{R}, \text { a.e. in } \Omega_{p} ; \\
\exists \pi^{\#}>0 ; & \left|K_{i j}(x, e)-K_{i j}(y, e)\right| \leq \pi^{\#}|x-y|, \quad \forall e \in \mathbb{R}, x, y \in \bar{\Omega}_{p} ; \\
\exists L_{k}>0: & \left|K_{i j}\left(x, e_{1}\right)-K_{i j}\left(x, e_{2}\right)\right| \leq L_{k}\left|e_{1}-e_{2}\right|, \quad \forall e_{1}, e_{2} \in \mathbb{R}, x \in \bar{\Omega}_{p} .
\end{array}
$$

Then there exists at least one weak solution $(\mathbf{u}, p, h, \theta) \in \mathbf{H}_{\Gamma_{f}}^{1}\left(\Omega_{f}\right) \times L^{2}\left(\Omega_{f}\right) \times$ $H_{\Gamma_{p}}^{1}\left(\Omega_{p}\right) \times W_{\gamma, \Gamma_{f}}^{1, r}(\Omega)$ in accordance with Definition 3.9 .

REMARK 3.11. Suppose that all above assumptions of Theorem 3.6 are fulfilled except (3.12)-(3.13). It suffices to consider the coefficient $\mathbf{K}$ as a Carathéodory function fulfilling (3.19) and

$$
\exists \pi^{\#}>0: \quad \sum_{i, j=1}^{n}\left|K_{i j}(\cdot, e)\right| \leq \pi^{\#} \quad \forall e \in \mathbb{R}, \text { a.e. in } \Omega_{p},
$$

in order to show that the solvability of the BJS-SDF problem remains true in the following version:

$$
\begin{aligned}
& \int_{\Omega_{f}} \mu(\theta) D \mathbf{u}: D \mathbf{v} d x+\int_{\Omega_{f}} \lambda(\theta) \nabla \cdot \mathbf{u} \nabla \cdot \mathbf{v} d x+\int_{\Omega_{p}}(\mathbf{K}(\theta) \nabla h) \cdot \nabla v d x \\
& \quad+\int_{\Gamma} h \mathbf{v} \cdot \mathbf{n} d s-\int_{\Gamma} \mathbf{u} \cdot \mathbf{n} v d s+\int_{\Gamma} \beta(\theta) \mathbf{u} \cdot \mathbf{v} d s \\
& =\int_{\Omega_{f}} p \nabla \cdot \mathbf{v} d x+\int_{\Omega_{f}} \mathbf{f}(\theta) \cdot \mathbf{v} d x, \quad \forall(\mathbf{v}, v) \in \mathbf{H}_{\Gamma_{f}}^{1}\left(\Omega_{f}\right) \times H_{\Gamma_{p}}^{1}\left(\Omega_{p}\right),
\end{aligned}
$$

with (3.17)-(3.18) kept unchanged.

The existence of weak solutions is based on the Tychonoff extension to weak topologies of the Schauder fixed point theorem [19].

THEOREM 3.12. Let $K$ be a nonempty compact convex subset of a locally convex space $X$. Let $\mathcal{L}: K \rightarrow K$ be a continuous operator. Then $\mathcal{L}$ has at least one fixed point.

In order to clarify the variational formulation (3.9), we multiply (2.2) and (2.5) by suitable test functions, integrate over each domain and apply Green's formula. From (2.2), we find

$$
\int_{\Omega_{f}} \tau: D \mathbf{v} d x-\left\langle\tau_{T}+\tau_{N} \mathbf{n}, \mathbf{v}\right\rangle_{\Gamma}=\int_{\Omega_{f}} p \nabla \cdot \mathbf{v} d x-\langle\mathbf{v} \cdot \mathbf{n}, p\rangle_{\Gamma}+\int_{\Omega_{f}} \mathbf{f}(\theta) \cdot \mathbf{v} d x
$$

for all $\mathbf{v} \in \mathbf{H}_{\Gamma_{f}}^{1}\left(\Omega_{f}\right)$, provided that $p$ has sufficiently regularity. From (2.5), we find

$$
\int_{\Omega_{p}} \mu(\theta) \Pi^{-1}(\theta) \mathbf{u}_{D} \cdot \mathbf{v} d x=\langle\mathbf{v} \cdot \mathbf{n}, p\rangle_{\Gamma}+\int_{\Omega_{p}} \mathbf{g}(\theta) \cdot \mathbf{v} d x,
$$


for all $\mathbf{v} \in \mathbf{H}$. Now, summing the above equalities and applying (2.16) and (2.20) we obtain (3.9).

Finally, in order to clarify the variational formulation (3.16), from (2.7) (using (2.6)) we find

$$
\int_{\Omega_{p}}(\mathbf{K}(\theta) \nabla h) \cdot \nabla v d x=\int_{\Gamma} \mathbf{u}_{D} \cdot \mathbf{n} v d s
$$

for all $v \in H_{\Gamma_{p}}^{1}\left(\Omega_{p}\right)$. Next, summing the above equality with (3.22) and using (2.16)(2.19) we arrive at (3.16).

We emphasize that $\mathbf{K}(\theta) \in \mathbf{L}^{\infty}\left(\Omega_{p}\right)$ and $h \in H^{1}\left(\Omega_{p}\right)$ imply that $\mathbf{K}(\theta) \nabla h \in \mathbf{L}^{2}\left(\Omega_{p}\right)$, which together with (2.6) and (2.7) implies $\mathbf{K}(\theta) \nabla h \in \mathbf{H}\left(\operatorname{div}, \Omega_{p}\right)$. It is known that the vectors of $\mathbf{H}\left(\operatorname{div}, \Omega_{p}\right)$ admit a well defined normal trace on $\partial \Omega_{p}$, that is, the normal trace operator $\mathbf{v} \mapsto v_{N}=\left.\mathbf{v} \cdot \mathbf{n}\right|_{\partial \Omega_{p}}$ applies $\mathbf{H}\left(\operatorname{div}, \Omega_{p}\right)$ onto $H^{-1 / 2}\left(\partial \Omega_{p}\right)=$ $\left(H^{1 / 2}\left(\partial \Omega_{p}\right)\right)^{\prime}$ [6. pp. 91-92] and is surjective from $\left\{\mathbf{v} \in \mathbf{H}\left(\operatorname{div}, \Omega_{p}\right): \nabla \cdot \mathbf{v}=0\right\}$ onto $\left\{v^{*} \in H^{-1 / 2}\left(\partial \Omega_{p}\right):\left\langle v^{*}, 1\right\rangle=0\right\}$ 6, pp. 92-93]. Even if we suppose $\Omega_{p} \in C^{1,1}$, that is, each component $n_{i}$ is Lipschitz continuous with uniform constant on $\partial \Omega_{p}$ (see Remark 3.1), the decomposition $\mathbf{v}=v_{N} \mathbf{n}+\mathbf{v}_{T}$ is an isomorphism from $\mathbf{H}^{1 / 2}\left(\partial \Omega_{p}\right)$ onto $H^{1 / 2}\left(\partial \Omega_{p}\right) \times \mathbf{H}_{T}^{1 / 2}\left(\partial \Omega_{p}\right)$, where the tangential space $\mathbf{H}_{T}^{1 / 2}\left(\partial \Omega_{p}\right)$ is the closed subspace of $\mathbf{H}^{1 / 2}\left(\partial \Omega_{p}\right)$ such that

$$
\mathbf{H}^{1 / 2}\left(\partial \Omega_{p}\right)=\mathbf{H}_{T}^{1 / 2}\left(\partial \Omega_{p}\right) \oplus\left\{\mathbf{v} \in \mathbf{H}^{1 / 2}\left(\partial \Omega_{p}\right): \mathbf{v}=v \mathbf{n}, v \in H^{1 / 2}\left(\partial \Omega_{p}\right)\right\} .
$$

Hence the arbitrariness of the element $\mathbf{v}_{T}$ implies that $\mathbf{v}$ is ambiguously defined as a function on $v$ only. Indeed, the above considerations are not sufficient to justify that $\mathbf{K}(\theta) \nabla h \in\left(\mathbf{H}_{00}^{1 / 2}(\Gamma)\right)^{\prime}$. To see this, we appeal to (2.19). Since the distribution $\tau \cdot \mathbf{n}$ is well-defined on $\Gamma$ as an element of $\left(\mathbf{H}_{00}^{1 / 2}(\Gamma)\right)^{\prime}$, then if $\Omega \in C^{1,1}, \tau_{N} \in\left(H_{00}^{1 / 2}(\Gamma)\right)^{\prime}$ and $\tau_{T} \in\left(\mathbf{H}_{00}^{1 / 2}(\Gamma)\right)^{\prime}$. Therefore, the ambiguity in the definition of $\mathbf{K}(\theta) \nabla h$ on $\Gamma$ is removed when we apply the property of product of functions in Sobolev spaces (see [20], for instance) for $\mathbf{u}_{S} \in \mathbf{H}_{00}^{1 / 2}(\Gamma)$ and the Nemytskii operator $\beta(\theta) \in C^{0,1}(\Gamma)$.

\section{Existence of unique auxiliary temperatures}

In this Section, we establish several existence results of unique auxiliary temperatures depending on the behavior of the domain and the leading coefficient.

LEMmA 4.1. Suppose that $(\mathbf{w}, \xi) \in \mathbf{V} \times H_{\gamma, \Gamma_{f}}^{1}(\Omega)$ and the assumptions (3.5) and (3.15) are fulfilled. If $n \leq 4$, then there exists a unique $\theta \in W_{\gamma, \Gamma_{f}}^{1,2}(\Omega)$ which satisfies

$$
\int_{\Omega}(\mathbb{K}(\xi) \nabla \theta) \cdot \nabla \psi d x=-\int_{\Omega} \varkappa(\xi) \mathbf{w} \cdot \nabla \xi \psi d x, \quad \forall \psi \in W_{\gamma, \Gamma_{f}}^{1,2}(\Omega) .
$$

For the Neumann problem $(\gamma=1)$, the uniqueness of a solution is established modulo a constant provided that the compatibility condition

$$
\int_{\Omega} \varkappa(\xi) \mathbf{w} \cdot \nabla \xi d x=0
$$

is satisfied. Moreover,

$$
\|\nabla \theta\|_{2, \Omega} \leq \varkappa^{\#} C_{\gamma}\|\mathbf{w}\|_{4, \Omega}\|\nabla \xi\|_{2, \Omega}
$$


with $C_{\gamma}$ denoting the Poincaré constant in accordance with the appropriate class ( $\gamma=$ $0,1)$.

Proof. For $(\mathbf{w}, \xi) \in \mathbf{V} \times H_{\gamma, \Gamma_{f}}^{1}(\Omega)$, by (3.5) we get $\varkappa(\xi) \mathbf{w} \cdot \nabla \xi \in L^{4 / 3}(\Omega) \hookrightarrow$ $\left(H_{\gamma, \Gamma_{f}}^{1}(\Omega)\right)^{\prime}$ if $n \leq 4$. Thus, the existence of a unique weak solution $\theta=\theta(\mathbf{w}, \xi) \in$ $H_{\gamma, \Gamma_{f}}^{1}(\Omega)$ is a consequence of the Lax-Milgram Lemma (see [6], for instance). The estimate (4.3) easily holds by taking $\psi=\theta$ as a test function in (4.1).

Proposition 4.2. Under the conditions of Lemma 4.1. for $n=2,3$, each solution $\theta \in H_{\gamma, \Gamma_{f}}^{1}(\Omega)$ satisfies $\theta \in W^{1,2+\varepsilon}(\Omega)$, for some $0<\varepsilon<1$, with the estimate

$$
\|\theta\|_{1,2+\varepsilon, \Omega} \leq C\left(\beta_{\#} / \beta^{\#}, \Omega\right) \varkappa^{\#}\|\mathbf{w}\|_{4, \Omega}\|\xi\|_{1,2, \Omega} .
$$

Proof. Let $\theta \in H_{\gamma, \Gamma_{f}}^{1}(\Omega)$ be any solution to the problem (4.1). Since we have $\varkappa(\xi) \mathbf{w} \cdot \nabla \xi \in L^{3 / 2}(\Omega) \hookrightarrow\left(W^{1, r^{\prime}}(\Omega)\right)^{\prime}$ for all $1<r^{\prime}<2$ whenever $n=2,3$, using the $W^{1,2+\varepsilon}$ regularity for solutions to second order elliptic differential equations with bounded measurable coefficients [21, 26, we obtain $\theta \in W^{1,2+\varepsilon}(\Omega)$ for some $\varepsilon>0$ depending on the boundary such that $2+\varepsilon \leq r$.

REMARK 4.3. If $n=2, \mathbf{w} \in \mathbf{H}_{\Gamma_{f}}^{1}(\Omega) \hookrightarrow L^{2(2+\varepsilon) / \varepsilon}(\Omega)$ for $\varepsilon>0$ in accordance with Proposition 4.2 Then, $\alpha(\xi) \mathbf{w} \cdot \nabla \xi \in L^{2}(\Omega)$ by $\nabla \xi \in L^{2+\varepsilon}(\Omega)$.

In order to proceed with the $3 \mathrm{D}$ proof, we recall two regularity results, in a simplified version sufficient for our claim, from the regularity theory for the Neumann problem of a second-order elliptic equation with the leading coefficient matrices having small partially BMO semi-norms and the boundary of the domain is either locally the graph of a Lipschitz continuous function with a small Lipschitz constant [14, 15] or is Reifenberg flat 7 .

Theorem 4.4. Let $\Omega$ be a bounded domain. Suppose $\mathbb{A}=\left[A_{i j}\right] \in \mathbf{L}^{\infty}(\Omega)$ satisfy $0<$ $A_{\#} \leq A_{i j} \leq A^{\#}$ a.e. in $\Omega$. Given $r>2$ there exist small positive constants $\delta$ and $\iota$ such that under one of the assumptions (1 or 2) of Theorem 3.7, for each $\mathbf{f} \in \mathbf{L}^{r}(\Omega)$, the solvability of the Neumann problem

$$
-\nabla \cdot(\mathbb{A} \nabla u)=\nabla \cdot \mathbf{f} \text { in } \Omega, \quad(\mathbb{A} \nabla u+\mathbf{f}) \cdot \mathbf{n}=0 \text { on } \partial \Omega
$$

holds and its solution $u \in H^{1}(\Omega)$ is unique up to a constant, and is actually in $\mathbf{W}^{1, r}(\Omega)$ with the estimate

$$
\|\nabla u\|_{r, \Omega} \leq N\|\mathbf{f}\|_{r, \Omega} .
$$

Proposition 4.5. Let $n=3$. Suppose that the conditions of Lemma 4.1 hold. In addition, if $\xi \in W^{1,4}(\Omega)$, then there exist small positive constants $\delta$ and $\iota$ such that under one of the assumptions (1 or 2) of Theorem 3.7, any solution $\theta \in H^{1}(\Omega)$ of (4.1) with $\gamma=1$ belongs to $W^{1,4}(\Omega)$ and satisfies the estimate

$$
\|\theta\|_{1,4, \Omega} \leq N C_{S} C_{B} \varkappa^{\#}\|\mathbf{w}\|_{6, \Omega}\|\xi\|_{1,4, \Omega} .
$$

Proof. Let $(\mathbf{w}, \xi) \in \mathbf{V} \times W^{1,4}(\Omega)$, and $\theta \in H^{1}(\Omega)$ be any solution to the problem (4.1) with $\gamma=1$. Since we have $g=\varkappa(\xi) \mathbf{w} \cdot \nabla \xi \in L^{2}(\Omega)$ satisfying (4.2), there exists $\mathbf{f} \in \mathbf{W}_{0}^{1,2}(\Omega)$ such that $\operatorname{div} \mathbf{f}=g$ with the estimate

$$
\|\mathbf{f}\|_{4, \Omega} \leq C_{S}\|\mathbf{f}\|_{1,2, \Omega} \leq C_{S} C_{B}\|g\|_{2, \Omega},
$$

where $C_{S}$ and $C_{B}$ stand for the Sobolev and Bogovskii constants, respectively. Therefore, Theorem 4.4 ensures the claim. 


\section{Existence of a unique auxiliary constrained velocity}

Let $(\mathbf{w}, \xi) \in \mathbf{V} \times W_{\gamma, \Gamma_{f}}^{1, n+\varepsilon}(\Omega)$ and the corresponding auxiliary temperature $\theta \in$ $W_{\gamma, \Gamma_{f}}^{1, n+\varepsilon}(\Omega)$ be in accordance with Section 4 The next lemma establishes the Ladyzhenskaya-Babuska-Brezzi condition, often known as the inf-sup condition, in $\mathbf{V} \times Q$, whose proof can be found in [28, Lemma 3.2].

Lemma 5.1. The bilinear functional $b: \mathbf{V} \times Q \rightarrow \mathbb{R}$ defined as

$$
b(\mathbf{v}, q)=\int_{\Omega} q \nabla \cdot \mathbf{v} d x, \quad \forall(\mathbf{v}, q) \in \mathbf{V} \times Q,
$$

is continuous and satisfies the Ladyzhenskaya-Babuska-Brezzi condition

$$
\exists b_{0}>0: \quad \inf _{q \in Q \backslash\{0\} \mathbf{v} \in \mathbf{V} \backslash\{\mathbf{0}\}} \max _{0} b(\mathbf{v}, q) \geq b_{0} .
$$

Lemma 5.2. The bilinear functional $a: \mathbf{V} \times \mathbf{V} \rightarrow \mathbb{R}$ defined as

$$
\begin{aligned}
a\left(\left(\mathbf{u}_{S}, \mathbf{u}_{D}\right),\left(\mathbf{v}_{f}, \mathbf{v}_{p}\right)\right)= & \int_{\Omega_{f}} \mu(\theta) D \mathbf{u}_{S}: D \mathbf{v}_{f} d x+\int_{\Omega_{f}} \lambda(\theta) \nabla \cdot \mathbf{u}_{S} \nabla \cdot \mathbf{v}_{f} d x \\
& +\int_{\Omega_{p}} \mu(\theta) \Pi^{-1}(\theta) \mathbf{u}_{D} \cdot \mathbf{v}_{p} d x+\int_{\Gamma} \beta(\theta) \mathbf{u}_{S} \cdot \mathbf{v}_{f} d s
\end{aligned}
$$

for all $\left(\mathbf{u}_{S}, \mathbf{u}_{D}\right),\left(\mathbf{v}_{f}, \mathbf{v}_{p}\right) \in \mathbf{V}$, is continuous and coercive on the constraint set KerB, where $B \in \mathcal{L}\left(\mathbf{V}, Q^{\prime}\right)$ is the operator associated to the bilinear form $b(\cdot, \cdot): \mathbf{V} \times Q \rightarrow \mathbb{R}$ provided by the Riesz Representation Theorem. The coercivity constant is given by $a_{0}=\mu_{\#}\left(\pi_{\#}+(n-1) / n\right)$.

Proof. The continuity results from the boundedness property:

$$
\begin{array}{r}
\left|a\left(\left(\mathbf{u}_{S}, \mathbf{u}_{D}\right),\left(\mathbf{v}_{f}, \mathbf{v}_{p}\right)\right)\right| \leq\left(\left(\mu^{\#}+\lambda^{\#}+\beta^{\#}\right)\left\|\mathbf{u}_{S}\right\|_{\mathbf{H}^{1}\left(\Omega_{f}\right)}+\mu^{\#} \pi^{\#}\left\|\mathbf{u}_{D}\right\|_{\mathbf{H}}\right) \\
\times\left(\left\|\mathbf{v}_{f}\right\|_{\mathbf{H}^{1}\left(\Omega_{f}\right)}+\left\|\mathbf{v}_{p}\right\|_{\mathbf{H}}\right) .
\end{array}
$$

From $n \lambda(\theta)+\mu(\theta) \geq 0$ and (3.15), we have

$$
a\left(\left(\mathbf{u}_{S}, \mathbf{u}_{D}\right),\left(\mathbf{u}_{S}, \mathbf{u}_{D}\right)\right) \geq \int_{\Omega_{f}} \mu(\theta)\left(\left|D \mathbf{u}_{S}\right|^{2}-\frac{1}{n}\left(\nabla \cdot \mathbf{u}_{S}\right)^{2}\right) d x+\mu_{\#} \pi_{\#} \int_{\Omega_{p}}\left|\mathbf{u}_{D}\right|^{2} d x
$$

Using the fact that $(\nabla \cdot \mathbf{u})^{2} \leq|D \mathbf{u}|^{2}$ and (2.4) , it follows that

$$
a\left(\left(\mathbf{u}_{S}, \mathbf{u}_{D}\right),\left(\mathbf{u}_{S}, \mathbf{u}_{D}\right)\right) \geq \frac{(n-1)}{n} \mu_{\#}\left\|D \mathbf{u}_{S}\right\|_{2, \Omega_{f}}^{2}+\mu_{\#} \pi_{\#}\left\|\mathbf{u}_{D}\right\|_{\mathbf{H}}^{2} .
$$

Therefore, the $\mathbf{V}$-coercivity holds with $a_{0}$.

REMARK 5.3. The bilinear form

$$
a_{p}(\mathbf{u}, \mathbf{v})=\int_{\Omega_{p}} \mu(\theta) \Pi^{-1}(\theta) \mathbf{u} \cdot \mathbf{v} d x, \quad \forall \mathbf{u}, \mathbf{v} \in \mathbf{H}\left(\operatorname{div}, \Omega_{p}\right),
$$

is coercive on the kernel of the divergence operator, defined from $\mathbf{H}\left(\operatorname{div}, \Omega_{p}\right)$ into $L^{2}\left(\Omega_{p}\right)$, although not on $\mathbf{H}\left(\operatorname{div}, \Omega_{p}\right)$.

Then we are in a position to establish the following existence result. 
Proposition 5.4. There exists a unique solution $(\mathbf{u}, p) \in \mathbf{V} \times Q$ to the problem

$$
\begin{aligned}
a(\mathbf{u}, \mathbf{v})+b(\mathbf{v}, p) & =F(\mathbf{v}), \quad \forall \mathbf{v}=\left(\mathbf{v}_{f}, \mathbf{v}_{p}\right) \in \mathbf{V} ; \\
b(\mathbf{u}, q) & =G(q), \quad \forall q=\left(q_{f}, q_{p}\right) \in Q,
\end{aligned}
$$

with

$$
\begin{aligned}
& F(\mathbf{v})=\int_{\Omega_{f}} \mathbf{f}(\theta) \cdot \mathbf{v}_{f} d x+\int_{\Omega_{p}} \mathbf{g}(\theta) \cdot \mathbf{v}_{p} d x \\
& G(q)=\int_{\Omega_{f}} \alpha(\theta) \mathbf{w}_{S} \cdot \nabla \theta q d x
\end{aligned}
$$

Moreover, the following estimates hold:

$$
\begin{aligned}
\|\mathbf{u}\|_{\mathbf{v}} & \leq \frac{1}{a_{0}}\left(f^{\#}\left(\|\theta\|_{2, \Omega_{f}}+|\Omega|^{1 / 2}\right)+\rho^{\#} g\right)+\frac{\alpha^{\#}}{b_{0}}\left(\frac{\|a\|}{a_{0}}+1\right)\|\mathbf{w} \cdot \nabla \theta\|_{2, \Omega_{f}} \\
\|p\|_{Q} & \leq \frac{1}{b_{0}}\left(\frac{\|a\|}{a_{0}}+1\right)\left(f^{\#}\left(\|\theta\|_{2, \Omega_{f}}+|\Omega|^{1 / 2}\right)+\rho^{\#} g+\frac{\alpha^{\#}}{b_{0}}\|\mathbf{w} \cdot \nabla \theta\|_{2, \Omega_{f}}\right),
\end{aligned}
$$

with $\|a\|$ denoting the constant given by $\mu^{\#}\left(1+1 / \pi^{\#}\right)+n \lambda^{\#}+\beta^{\#}$.

Proof. The abstract theory on the quadratic problems under linear constraints [6. Theorem 1.1, p. 42] may be applied by ensuring that the linear form $a(\cdot, \cdot)$ is continuous on $\mathbf{V} \times \mathbf{V}$ and it is invertible on $\operatorname{Ker} B$, that is, that it satisfies the coercivity conditions

$$
\begin{aligned}
& \inf _{\mathbf{u} \in \operatorname{Ker} B \backslash\{0\} \mathbf{v} \in \operatorname{Ker} B \backslash\{\mathbf{0}\}} \frac{a(\mathbf{u}, \mathbf{v})}{\|\mathbf{u}\|_{\mathbf{v}}\|\mathbf{v}\|_{\mathbf{v}}} \geq a_{0} ; \\
& \inf _{\mathbf{v} \in \operatorname{Ker} B \backslash\{0\} \mathbf{u} \in \operatorname{Ker} B \backslash\{\mathbf{0}\}} \frac{a(\mathbf{u}, \mathbf{v})}{\|\mathbf{u}\|_{\mathbf{v}}\|\mathbf{v}\|_{\mathbf{v}}} \geq a_{0},
\end{aligned}
$$

and the linear form $b(\cdot, \cdot)$ is continuous on $\mathbf{V} \times Q$ and the range of the associated operator $B$ is closed in $Q^{\prime}$, that is, it satisfies the inf-sup condition

$$
\inf _{q \in\left(Q / \operatorname{Ker} B^{t}\right) \backslash\{0\} \mathbf{v} \in \mathbf{V} \backslash\{\mathbf{0}\}} \max _{\|\mathbf{v}\| \mathbf{v}\|q\|_{Q / \operatorname{Ker} B^{t}}} \geq b_{0},
$$

where $B^{t}$ is the transpose of $B$. These conditions are exactly Lemmas 5.1 and 5.2 by the symmetry of $a$. In virtue of Remark 4.3 and $\operatorname{Ker} B^{t}=\{0\}, G \in \operatorname{Im} B=Q^{\prime}$.

Since $F \in \mathbf{V}^{\prime}$, the existence of a unique $(\mathbf{u}, p) \in \mathbf{V} \times Q$ solution of (5.2)-(5.3) is guaranteed.

\section{The Beavers-Joseph-Saffman-Stokes-Darcy-Fourier problem}

In this section, we show simultaneously the proofs of Theorems 3.6 and 3.7 via the fixed point argument (cf. Theorem 3.12).

Proof of Theorems 3.6 and 3.7 .

Let $\varepsilon$ be the constant depending on the character of the domain in accordance with Proposition 4.2 if $n=2$, or $\varepsilon=1$ if $n=3$. Set the Banach space $X=\mathbf{V} \times W_{\gamma, \Gamma_{f}}^{1, n+\varepsilon}(\Omega)$, endowed with the product of weak topologies so that $X$ becomes a locally convex Hausdorff topological vector space. Let $K$ be the closed ball

$$
K=\left\{(\mathbf{w}, \xi) \in X:\|\mathbf{w}\|_{\mathbf{v}} \leq \frac{1}{C_{1}},\|\xi\|_{1, n+\varepsilon, \Omega} \leq \frac{a_{0}}{a_{0} C_{2}+f^{\#} C_{1}},\right.
$$

and (4.2) holds if $\gamma=1\}$, 
with

$$
\begin{aligned}
& C_{1}= \begin{cases}C\left(\beta_{\#} / \beta^{\#}, \Omega\right) \varkappa^{\#} & \text { if } n=2, \\
N C_{S} C_{B} \varkappa^{\#} & \text { if } n=3,\end{cases} \\
& C_{2}=\frac{1}{a_{0}}\left(f^{\#}|\Omega|^{1 / 2}+\rho^{\#} g\right)+\frac{\alpha^{\#}}{b_{0}}\left(\frac{\|a\|}{a_{0}}+1\right)
\end{aligned}
$$

according to the bound constants from Propositions 4.2 4.5, and 5.4. The ball $K$ is a nonempty weakly sequential compact convex subset of $X$.

For each $(\mathbf{w}, \xi) \in K$, the mapping $\mathcal{L}$ is well defined as

$$
\mathcal{L}(\mathbf{w}, \xi)=(\mathbf{u}, \theta)
$$

where $(\mathbf{u}, \theta)$ is the unique solution in accordance with Sections 4 and 5 . For $\gamma=1$, we take in the set of temperature auxiliary solutions the unique $\theta \in H^{1}(\Omega)$ such that

$$
\int_{\Omega} \varkappa(\theta) \mathbf{u} \cdot \nabla \theta d x=0
$$

Notice that the existence of an auxiliary pressure $p$ is the unique Lagrange multiplier associated to the constraint (5.3).

In order to apply Theorem 3.12, let us first show that for each $(\mathbf{w}, \xi) \in K$ we have $\mathcal{L}(\mathbf{w}, \xi) \in K$. Considering that $(\mathbf{w}, \xi) \in K$, from Propositions 4.2 and 4.5 we find

$$
\|\nabla \theta\|_{n+\varepsilon, \Omega} \leq \frac{a_{0}}{a_{0} C_{2}+f^{\#} C_{1}},
$$

and therefore (5.4) implies

$$
\|\mathbf{u}\| \mathbf{v} \leq \frac{f^{\#}}{a_{0}}\|\theta\|_{2, \Omega_{f}}+C_{2}\|\mathbf{w}\|_{2(n+\varepsilon) /(n+\varepsilon-2), \Omega_{f}}\|\nabla \theta\|_{n+\varepsilon, \Omega_{f}} \leq 1 / C_{1}
$$

Next, let us show that the operator $\mathcal{L}$ is continuous in $K$ for the weak topology. Let $\left\{\left(\mathbf{w}_{m}, \xi_{m}\right)\right\}_{m \in \mathbb{N}}$ be a sequence in $K$ satisfying

$$
\mathbf{w}_{m} \rightarrow \mathbf{w} \text { in } \mathbf{V}, \quad \xi_{m} \rightarrow \xi \text { in } W^{n+\varepsilon}(\Omega),
$$

and $\left(\mathbf{u}_{m}, \theta_{m}\right)=\mathcal{L}\left(\mathbf{w}_{m}, \xi_{m}\right)$ for each $m \in \mathbb{N}$. Applying the Rellich-Kondrakov embeddings $\mathbf{V} \hookrightarrow \hookrightarrow \mathbf{L}^{n}(\Omega)$ and $W^{1, n+\varepsilon}(\Omega) \hookrightarrow \hookrightarrow L^{1}(\Omega)$ we get

$$
\mathbf{w}_{m} \rightarrow \mathbf{w} \text { in } \mathbf{L}^{n}(\Omega), \quad \xi_{m} \rightarrow \xi \text { a.e. in } \Omega .
$$

From the estimate (6.2) we can extract a subsequence, still denoted by $\theta_{m}$, such that

$$
\theta_{m} \rightarrow \theta \text { in } W^{1, n+\varepsilon}(\Omega) .
$$

Thanks to the continuity of the Nemytskii operators $\mathbb{K}$ and $\varkappa$ on their second argument, the variational equality, for all $\psi \in W_{\gamma, \Gamma_{f}}^{1,(n+\varepsilon)^{\prime}}(\Omega) \hookrightarrow L^{n(n+\varepsilon) /(n(n+\varepsilon)-2 n-\varepsilon)}(\Omega)$,

$$
\int_{\Omega}\left(\mathbb{K}\left(\xi_{m}\right) \nabla \theta_{m}\right) \cdot \nabla \psi d x=\int_{\Omega} \varkappa\left(\xi_{m}\right) \mathbf{w}_{m} \cdot \nabla \xi_{m} \psi d x
$$

clearly passes to the limit as $m$ tends to infinity, proving (4.1). 
On the other hand, the solutions $\mathbf{u}_{m}=\mathbf{u}\left(\mathbf{w}_{m}, \theta_{m}\right)$ and $p_{m}=p\left(\mathbf{w}_{m}, \theta_{m}\right)$ satisfy (5.4) and (5.5), respectively, so we can extract a subsequence, still denoted by $\left(\mathbf{u}_{m}, p_{m}\right)$, such that

$$
\mathbf{u}_{m} \rightarrow \mathbf{u} \text { in } \mathbf{V}, \quad p_{m} \rightarrow p \text { in } L^{2}(\Omega) .
$$

Thus we pass to the limit as $m$ tends to infinity in $(5.2) m-(5.3) m$ and conclude that $(\mathbf{u}, \theta)=\mathcal{L}(\mathbf{w}, \xi)$, since $\alpha\left(\theta_{m}\right) \mathbf{w}_{m} \cdot \nabla \theta_{m}$ converges weakly to $\alpha(\theta) \mathbf{w} \cdot \nabla \theta$ in $L^{2}\left(\Omega_{f}\right)$.

In conclusion, Theorem 3.12 guarantees the existence of a fixed point $(\mathbf{u}, \theta)=$ $\mathcal{L}(\mathbf{u}, \theta)$, that is $(\mathbf{u}, p, \theta)$ is in the conditions of Theorem 3.6 and 3.7 Then, the proof of Theorem 3.6 and 3.7 is concluded.

7. Existence of a unique auxiliary velocity-head-temperature triple

Let $(\mathbf{w}, \varphi, \xi) \in \mathbf{H}_{\Gamma_{f}}^{1}\left(\Omega_{f}\right) \times W^{2,2}\left(\Omega_{p}\right) \times W_{\gamma, \Gamma_{f}}^{1,2+\varepsilon}(\Omega)$ and the corresponding auxiliary temperature $\theta \in W_{\gamma, \Gamma_{f}}^{1,2+\varepsilon}(\Omega)$, in analogy with Section 4 , satisfy

$$
\|\theta\|_{1,2+\varepsilon, \Omega} \leq C_{1}\left(\|\mathbf{w} \cdot \nabla \xi\|_{2, \Omega_{f}}+\|(\mathbf{K} \nabla \varphi) \cdot \nabla \xi\|_{2, \Omega_{p}}\right)
$$

with $C_{1}$ being the constant given in (6.1).

The first existence result goes to the piezometric head in a $n$-dimensional space.

Proposition 7.1. Suppose that the assumptions (3.20)-(3.21) are fulfilled. For each $\theta \in W^{1, n+\varepsilon}(\Omega)$, there exists a unique (modulo a constant) strong solution $h \in H^{2}\left(\Omega_{p}\right)$ to the Neumann problem

$$
\begin{aligned}
& \nabla \cdot(\mathbf{K}(\theta) \nabla h)=0 \quad \text { in } \Omega_{p} ; \\
& -(\mathbf{K}(\theta) \nabla h) \cdot \mathbf{n}=\mathbf{w} \cdot \mathbf{n} \quad \text { on } \Gamma \text {; } \\
& (\mathbf{K}(\theta) \nabla h) \cdot \mathbf{n}_{p}=0 \quad \text { on } \Gamma_{p} \text {, }
\end{aligned}
$$

satisfying

$$
\|h\|_{2,2, \Omega_{p}} \leq A\|\mathbf{w}\|_{\mathbf{H}_{\Gamma_{f}}^{1}\left(\Omega_{f}\right)},
$$

where the positive constant $A$ only depends on $n, \pi_{\#}$, and the continuity constant of the embedding $\mathbf{H}_{\Gamma_{f}}^{1}\left(\Omega_{f}\right) \hookrightarrow \mathbf{H}_{00}^{1 / 2}(\Gamma)$.

Proof. By Remark 3.1 the Morrey-Sobolev embedding $W^{1, n+\varepsilon}\left(\Omega_{p}\right) \hookrightarrow$ $C^{0,1-n /(n+\varepsilon)}\left(\bar{\Omega}_{p}\right)$ holds and consequently we have $\theta \in C^{0,1-n /(n+\varepsilon)}\left(\bar{\Omega}_{p}\right)$. Hence the Nemytskii operator $\mathbf{K} \in C^{0, \varepsilon /(n+\varepsilon)}\left(\bar{\Omega}_{p}\right)$ satisfies the continuity condition

$$
\left|K_{i j}(x, \theta(x))-K_{i j}(y, \theta(y))\right| \leq\left(\pi^{\#}+L_{k} M\right)|x-y|^{\varepsilon /(n+\varepsilon)}, \quad \forall x, y \in \bar{\Omega}_{p}: \quad|x-y| \leq 1,
$$

where $M$ is the Hölder constant of $\theta$, and $\pi^{\#}$ and $L_{k}>0$ are the Lipschitz constants of $\mathbf{K}$ in accordance with the assumptions (3.20) and (3.21), respectively.

Thanks to the regularity theory for the boundary value problem (7.2)-(7.4) due to the Korn perturbation method [20, pp. 107-109], since $\mathbf{w} \in \mathbf{H}_{00}^{1 / 2}(\Gamma)$, the solution $h$ belongs to $W^{2,2}\left(\Omega_{p}\right)$. Moreover, $h$ satisfies the estimate (7.5).

The existence of a constraint Stokes velocity is analyzed in terms of a saddlepoint approach. Let us recall the definition of a saddle-point and its existence [17, Proposition 2.4, p. 164].

Definition 7.2. Let $V$ and $Z$ be two Banach spaces and $L: V \times Z \rightarrow \mathbb{R}$. We say that $(u, p)$ is a saddle-point of a differentiable $L$ if it satisfies

$$
\left\langle\frac{\partial L}{\partial v}(u, p), v\right\rangle=0, \quad \forall v \in V
$$




$$
\left\langle\frac{\partial L}{\partial q}(u, p), q\right\rangle=0, \quad \forall q \in Z
$$

where the symbol $\langle\cdot, \cdot\rangle$ denotes a generic duality pairing, not distinguished between scalar and vector fields.

Theorem 7.3. Let $V$ and $Z$ be two reflexive Banach spaces and $L: V \times Z \rightarrow \mathbb{R}$ be the Lagrangian functional such that

$$
\begin{array}{r}
\forall p \in Z, \quad u \mapsto L(u, p) \text { is convex and lower semicontinuous on } V ; \\
\exists p_{0} \in Z, \quad L\left(u, p_{0}\right) \rightarrow+\infty \text { as }\|u\| \rightarrow+\infty \text { on } V ; \\
\forall u \in V, \quad p \mapsto L(u, p) \text { is concave and upper semicontinuous on } Z ; \\
\exists u_{0} \in V, \quad L\left(u_{0}, p\right) \rightarrow-\infty \text { as }\|p\| \rightarrow+\infty \text { on } Z
\end{array}
$$

Then L has a saddle-point.

Let us set $V=\mathbf{H}_{\Gamma_{f}}^{1}\left(\Omega_{f}\right)$ and $Z=L^{2}\left(\Omega_{f}\right)$ and introduce the Lagrangian functional $L: V \times Z \rightarrow \mathbb{R}$ as

$$
L(\mathbf{v}, p)=\mathcal{F}(\mathbf{v})+\int_{\Omega_{f}} p \mathcal{Q}(\mathbf{v}) d x
$$

with $\mathcal{F}: \mathbf{H}_{\Gamma_{f}}^{1}\left(\Omega_{f}\right) \rightarrow \mathbb{R}$ representing the energy functional associated to the motion system

$$
\begin{aligned}
\mathcal{F}(\mathbf{v})= & \int_{\Omega_{f}}\left(\mu(\theta) \frac{|D \mathbf{v}|^{2}}{2}+\lambda(\theta) \frac{|\nabla \cdot \mathbf{v}|^{2}}{2}-\mathbf{f}(\theta) \cdot \mathbf{v}\right) d x \\
& +\int_{\Gamma} \beta(\theta) \frac{|\mathbf{v}|^{2}}{2} d s+\int_{\Gamma} \beta(\theta)(\mathbf{K}(\theta) \nabla h) \cdot \mathbf{v} d s+\int_{\Gamma} h \mathbf{v} \cdot \mathbf{n} d s
\end{aligned}
$$

and the constraint given by

$$
\mathcal{Q}(\mathbf{v})=\nabla \cdot \mathbf{v}-\alpha(\theta) \mathbf{w} \cdot \nabla \theta
$$

Let us verify the conditions stated at Theorem 7.3 . The condition (7.8) is a consequence of $\mathcal{F}$ being continuous and convex while $\mathcal{Q}$ is linear and continuous. For $p_{0}=0 \in Z$, the condition (7.9) is satisfied. The condition (7.10) is satisfied since $p \mapsto L(\mathbf{v}, p)$ is an affine continuous mapping. Finally in order to check (7.11), it suffices to choose $u_{0}=\mathbf{0} \in V$.

Then Theorem 7.3 guarantees the existence of a saddle-point $(\mathbf{u}, p)$ to $L$. This saddle-point is the required solution to Theorem 3.10, since the problem (7.6)-(7.7) is exactly (cf. [11])

$$
\begin{aligned}
& \int_{\Omega_{f}} \mu(\theta) D \mathbf{u}: D \mathbf{v} d x+\int_{\Omega_{f}} \lambda(\theta) \nabla \cdot \mathbf{u} \nabla \cdot \mathbf{v} d x+\int_{\Gamma} \beta(\theta)(\mathbf{u}+\mathbf{K}(\theta) \nabla h) \cdot \mathbf{v} d s+\int_{\Gamma} h \mathbf{v} \cdot \mathbf{n} d s \\
= & \int_{\Omega_{f}} p \nabla \cdot \mathbf{v} d x+\int_{\Omega_{f}} \mathbf{f}(\theta) \cdot \mathbf{v} d x, \quad \forall \mathbf{v} \in V ; \\
& \int_{\Omega_{f}} q \mathcal{Q}(\mathbf{u}) d x=0, \quad \forall q \in Q .
\end{aligned}
$$


To prove the uniqueness of $\mathbf{u}$ and $p$ (up to a constant), we make a standard argument by taking two solutions $\left(\mathbf{u}_{1}, p_{1}\right)$ and $\left(\mathbf{u}_{2}, p_{2}\right)$, subtracting their variational equalities, choosing $\overline{\mathbf{u}}=\mathbf{u}_{1}-\mathbf{u}_{2}$ and $\bar{p}=p_{1}-p_{2}$ as test functions, and obtain

$$
\int_{\Omega_{f}} \mu(\theta)|D \overline{\mathbf{u}}|^{2} d x+\int_{\Omega_{f}} \lambda(\theta)|\nabla \cdot \overline{\mathbf{u}}|^{2} d x+\int_{\Gamma} \beta(\theta)|\overline{\mathbf{u}}|^{2} d s=\int_{\Omega_{f}} \bar{p} \nabla \cdot \overline{\mathbf{u}} d x=0 .
$$

Therefore, we conclude that $\mathbf{u}_{1} \equiv \mathbf{u}_{2}$ and consequently $\nabla p_{1} \equiv \nabla p_{2}$.

Let us make the estimates satisfied by $(\mathbf{u}, p)$ explicit.

Lemma 7.4. Let $(\mathbf{w}, \mathbf{u}, p, h, \theta) \in\left(\mathbf{H}_{\Gamma_{f}}^{1}\left(\Omega_{f}\right)\right)^{2} \times L^{2}\left(\Omega_{f}\right) \times W^{2,2}\left(\Omega_{p}\right) \times W_{\gamma, \Gamma_{f}}^{1, n+\varepsilon}(\Omega)$ satisfy (7.12)-(7.13). Then the following estimates hold:

$$
\begin{aligned}
\|p\|_{2, \Omega_{f}} \leq & \left(\mu^{\#}+\lambda^{\#}\right)\|\mathbf{u}\|_{1,2, \Omega_{f}}+C_{f} f^{\#}\left(\|\theta\|_{2, \Omega_{f}}+|\Omega|^{1 / 2}\right) ; \\
\|\mathbf{u}\|_{1,2, \Omega_{f}} \leq \pi^{\#} & \sqrt{\frac{\beta^{\#}}{\nu}}\|h\|_{2,2, \Omega_{p}}+\frac{1}{\nu}\left(\|h\|_{2, \Gamma}+f^{\#}\left(\|\theta\|_{2, \Omega_{f}}+|\Omega|^{1 / 2}\right)\right) \\
& +\frac{\alpha^{\#}\left(\mu^{\#}+\lambda^{\#}\right)}{\nu}\|\mathbf{w} \cdot \nabla \theta\|_{2, \Omega_{f}} \\
& +\sqrt{\frac{C_{f} f^{\#}\left(\|\theta\|_{2, \Omega_{f}}+|\Omega|^{1 / 2}\right) \alpha^{\#}}{\nu}}\|\mathbf{w} \cdot \nabla \theta\|_{2, \Omega_{f}} .
\end{aligned}
$$

Proof. First, taking $q=\nabla \cdot \mathbf{u}$ as a test function in (7.13) and applying (3.4), it follows that

$$
\|\nabla \cdot \mathbf{u}\|_{2, \Omega_{f}} \leq \alpha^{\#}\|\mathbf{w} \cdot \nabla \theta\|_{2, \Omega_{f}} .
$$

Secondly, we take $\mathbf{v} \in \mathbf{H}_{0}^{1}\left(\Omega_{f}\right)$ such that $\operatorname{div} \mathbf{v}=p$ as a test function in (7.12). This existence follows, for instance, from the classical theory for the Dirichlet-Laplace problem provided that $p$ has mean value zero. Moreover, we have

$$
\|\mathbf{v}\|_{1,2, \Omega_{f}} \leq C_{f}\|p\|_{2, \Omega_{f}} .
$$

Then, using (3.1)-(3.2) and (3.7) we obtain (7.14).

Finally, we choose $\mathbf{v}=\mathbf{u}$ as a test function in (7.12). Proceeding as in Lemma 5.2 and applying (7.14), (7.16), and the Young inequality, we derive

$$
\begin{aligned}
\nu\|\mathbf{u}\|_{1,2, \Omega_{f}}^{2}+\|\sqrt{\beta(\theta)} \mathbf{u}\|_{2, \Gamma}^{2} \leq & \frac{1}{2} \| \\
& \sqrt{\beta(\theta)}(\mathbf{K}(\theta) \nabla h)\left\|_{2, \Gamma}^{2}+\frac{1}{2}\right\| \sqrt{\beta(\theta)} \mathbf{u} \|_{2, \Gamma}^{2} \\
& +\frac{\nu}{4}\|\mathbf{u}\|_{1,2, \Omega_{f}}^{2}+\frac{\left(\alpha^{\#}\left(\mu^{\#}+\lambda^{\#}\right)\right)^{2}}{\nu}\|\mathbf{w} \cdot \nabla \theta\|_{2, \Omega_{f}}^{2} \\
& +C_{f} f^{\#}\left(\|\theta\|_{2, \Omega_{f}}+|\Omega|^{1 / 2}\right) \alpha^{\#}\|\mathbf{w} \cdot \nabla \theta\|_{2, \Omega_{f}} \\
& +\frac{1}{\nu}\left(\|h\|_{2, \Gamma}+f^{\#}\left(\|\theta\|_{2, \Omega_{f}}+|\Omega|^{1 / 2}\right)\right)^{2}+\frac{\nu}{4}\|\mathbf{u}\|_{1,2, \Omega_{f}}^{2}
\end{aligned}
$$

with $\nu=C_{P}(n-1) \mu_{\#} / n, n>1$ and $C_{P}$ the Poincaré constant. Therefore we have (7.15), which concludes the proof. 


\section{The Beavers-Joseph-Stokes-Darcy-Fourier problem}

\section{Proof of Theorem 3.10 .}

In order to apply Theorem 3.12, we redefine $X=\mathbf{H}_{\Gamma_{f}}^{1}\left(\Omega_{f}\right) \times W^{2,2}\left(\Omega_{p}\right) \times$ $W_{\gamma, \Gamma_{f}}^{1,2+\varepsilon}(\Omega)$ and the closed ball $K$ by

$$
K=\left\{(\mathbf{w}, \varphi, \xi) \in X:\|\mathbf{w}\|_{1,2, \Omega_{f}} \leq \frac{1}{C_{3}},\|\varphi\|_{2,2, \Omega_{p}} \leq \frac{A}{C_{3}},\|\xi\|_{1,2+\varepsilon, \Omega} \leq R\right\},
$$

with

$$
\begin{array}{r}
C_{3}=\max \left\{1, C_{1}\left(1+\pi^{\#} A\right), \frac{1}{\nu}\left(A+f^{\#}\left(R+|\Omega|^{1 / 2}\right)+\alpha^{\#}\left(\mu^{\#}+\lambda^{\#}\right) R\right)\right. \\
\left.+\frac{1}{\sqrt{\nu}}\left(\pi^{\#} A \sqrt{\beta^{\#}}++\sqrt{C_{f} f^{\#} \alpha^{\#} R\left(R+|\Omega|^{1 / 2}\right)}\right)\right\}
\end{array}
$$

according to the bound constants from (17.1), (7.5), and (7.14)-(7.15). As in Section 6, if the Banach space $X$ is endowed with the product of weak topologies, then $X$ becomes a locally convex Hausdorff topological vector space and the ball $K$ is a nonempty weakly sequential compact convex subset of $X$. Now we set the mapping $\mathcal{L}$ defined as

$$
\mathcal{L}(\mathbf{w}, \varphi, \xi)=(\mathbf{u}, h, \theta),
$$

where $(\mathbf{u}, h, \theta)$ is the unique solution in accordance with Section 7 Notice that the existence of an auxiliary pressure $p$ is defined up to an additive constant.

The continuity of $\mathcal{L}$ follows by taking $\left(\mathbf{w}_{m}, \varphi_{m}, \xi_{m}\right)$ weakly convergent to $(\mathbf{w}, \varphi, \xi)$ in $X$ and the corresponding solutions $\left(\mathbf{u}_{m}, h_{m}, \theta_{m}\right)=\mathcal{L}\left(\mathbf{w}_{m}, \varphi_{m}, \xi_{m}\right)$ with Lagrange multipliers $p_{m}$ to satisfy the estimates (7.1), (7.5) and (7.14)-(7.15). Then, we can extract subsequences such that the following convergences hold for $r>2$ :

$$
\begin{aligned}
\mathbf{w}_{m} \rightarrow \mathbf{w}, & \mathbf{u}_{m} \rightarrow \mathbf{u} \text { in } \mathbf{H}^{1}\left(\Omega_{f}\right) \hookrightarrow \hookrightarrow \mathbf{L}^{2 r /(r-2)}\left(\Omega_{f}\right) ; \\
& p_{m} \rightarrow p \text { in } L^{2}\left(\Omega_{f}\right) ; \\
\varphi_{m} \rightarrow \varphi, & h_{m} \rightarrow h \text { in } H^{2}\left(\Omega_{p}\right) \hookrightarrow \hookrightarrow H^{1}(\Gamma) ; \\
\xi_{m} \rightarrow \xi, & \theta_{m} \rightarrow \theta \text { in } W^{1, r}(\Omega) \hookrightarrow \hookrightarrow C^{0,1-2 / r}(\bar{\Omega}) .
\end{aligned}
$$

Observing that the passage to the limit (as $m$ tends to infinity) into the variational equalities can be applied, we conclude that $(\mathbf{u}, h, \theta)=\mathcal{L}(\mathbf{w}, \varphi, \xi)$ with Lagrange multiplier $p$.

Finally, we see that $(\mathbf{u}, h, \theta)=\mathcal{L}(\mathbf{w}, \varphi, \xi) \in K$ for each $(\mathbf{w}, \varphi, \xi) \in K$. Take an arbitrary $(\mathbf{w}, \varphi, \xi) \in K$ and set $(\mathbf{u}, h, \theta)=\mathcal{L}(\mathbf{w}, \varphi, \xi)$ with Lagrange multiplier $p$. By (7.1) and (7.5) we get, respectively,

$$
\begin{aligned}
\|\theta\|_{1,2+\varepsilon, \Omega} & \leq C_{1}\left(1+\pi^{\#} A\right) R / C_{3} \leq R \\
\|h\|_{2,2, \Omega_{p}} & \leq A / C_{3} .
\end{aligned}
$$

From (7.14)-(7.15), it results that

$$
\begin{aligned}
\|\mathbf{u}\|_{1,2, \Omega_{f}} \leq \pi^{\#} A \sqrt{\frac{\beta^{\#}}{\nu}}+\frac{A}{\nu}+ & \frac{f^{\#}}{\nu}\left(R+|\Omega|^{1 / 2}\right)+\frac{\alpha^{\#}\left(\mu^{\#}+\lambda^{\#}\right) R}{\nu} \\
& +\sqrt{\frac{C_{f} f^{\#}\left(R+|\Omega|^{1 / 2}\right) \alpha^{\#} R}{\nu}} \leq \frac{1}{C_{3}} .
\end{aligned}
$$


In conclusion we can apply the fixed point argument (see Theorem 3.12) to ensure the existence of at least one required solution $(\mathbf{u}, p, h, \theta)$, which completes the proof of Theorem 3.10,

\section{REFERENCES}

[1] B. Alazmi and K. Vafai, Constant wall heat flux boundary conditions in porous media under local thermal non-equilibrium conditions, Int. J. Heat and Mass Trans., 45, 3071-3087, 2002.

[2] T. Arbocast and D.S. Brunson, A computational method for approximating a Darcy-Stokes system governing a vuggy porous medium, Comp. Geosci., 11(3), 207-218, 2007.

[3] S. Badia and R. Codina, Unified stabilized finite element formulations for the Stokes and the Darcy problems, SIAM J. Numer. Anal., 47, 1971-2000, 2009.

[4] M. Le Bars and M.G. Worster, Interfacial conditions between a pure fluid and a porous medium: Implications for binary alloy solidification, J. Fluid Mech., 550, 149-173, 2006.

[5] G.S. Beavers and D.D. Joseph, Boundary conditions at a naturally permeable wall, J. Fluid Mech., 30, 197-207, 1967.

[6] F. Brezzi and M. Fortin, Mixed and Hybrid Finite Element Methods, Springer-Verlag, New York Inc., 1991.

[7] S. Byun, H. Chen, M. Kim, and L. Wang, $L^{p}$ regularity theory for linear elliptic systems, Discrete Contin. Dyn. Syst., 18(1), 121-134, 2007.

[8] Y. Cao, M. Gunsburger, F. Hua, and X. Wang, Coupled Stokes-Darcy model with Beavers-Joseph interface boundary condition, Commun. Math. Sci., 8(1), 1-25, 2010.

[9] P. Celle, S. Drapier and J.M. Bergheau, Numerical modelling of liquid infusion into fibrous media undergoing compaction, Eur. J. Mech. - A/Solids, 27(4), 647-661, 2009.

[10] A. Cesmelioglu and B. Rivière, Analysis of time-dependent Navier-Stokes flow coupled with Darcy flow, J. Numer. Math., 16(4), 249-280, 2008.

[11] L. Consiglieri, The non-incompressibility on Navier-Stokes-Fourier fluids. The stationary case, J. Math. Fluid Mech., 12(3), 379-396, 2010.

[12] L. Consiglieri, Mathematical Analysis of Selected Problems from Fluid Thermomechanics: The $(p-q)$ Coupled Fluid-energy Systems, Lambert Academic Publishing, Saarbrücken, 2011.

[13] M. Discacciati, A. Quarteroni, and A. Valli, Robin-Robin domain decomposition methods for the Stokes-Darcy coupling, SIAM J. Numer. Anal., 45, 1246-1268, 2007.

[14] H. Dong and D. Kim, Elliptic equations in divergence form with partially BMO coefficients, Arch. Rat. Mech. Anal., 196(1), 25-70, 2010.

[15] H. Dong and D. Kim, $L^{p}$ solvability of divergence type parabolic and elliptic systems with partially BMO coefficients, Calc. Var. Part. Diff. Eqs., 40(3-4), 357-389, 2011.

[16] M. Ehrhardt, J. Fuhrmann, E. Holzbecher, and A. Linke, Mathematical modeling of channel porous layer interfaces in PEM fuel cells, in B. Davat B, Hissel D (eds.), Proceedings of FDFC2008 - Fundamentals and Developments of Fuel Cell Conference 2008, Nancy, France.

[17] I. Ekeland and R. Temam, Analyse Convexe et Problèmes Variationnels, Dunod et GauthierVillars, Paris, 1974.

[18] A. Faghri and L.C. Chow, Forced condensation in a tube with suction at the wall: Microgravitational application, J. Heat Trans., 110, 982-985, 1988.

[19] K. Fan, A generalization of Tychonoff's fixed point theorem, Math. Annal., 142, 305-310, 1961.

[20] P. Grisvard, Elliptic Problems in Nonsmooth Domains, Monographs and Studies in Mathematics, Pitman, Boston, 24, 1985.

[21] K. Gröger and J. Rehberg, Resolvent estimates in $W^{1,-p}$ for second order elliptic differential operators in case of mixed boundary conditions, Math. Annal. 285, 105-113, 1989.

[22] R. Haller-Dintelmann, H.C. Kaiser, and J. Rehberg, Elliptic model problems including mixed boundary conditions and material heterogeneities, J. Math. Pures Appl., 89, 25-48, 2008.

[23] A.A. Hill, Horizontal thermal convection in a porous medium, Continuum Mech. Thermodyn., $18,253-258,2006$

[24] C.C. Horng, T.S. Sheu, B.H. Hwang, C.J. Wan, and P.C. Liu, Temperature distribution in a porous medium subjected to solar radiative incidence and downward flow, WHAMPOA - An Interdisciplinary Journal, 50, 185-194, 2006.

[25] Y. Kagei, M. Rŭžička, and G. Thäter, Natural convection with dissipative heating, Commun. Math. Phys., 214, 287-313, 2000.

[26] C.E. Kenig and J. Pipher, The Neumann problem for elliptic equations with non-smooth coefficients, Invent. Math., 113, 447-509, 1993.

[27] W. Jäger and A. Mikelić, On the interface boundary condition of Beavers, Joseph, and Saffman, SIAM J. Appl. Math., 60, 1111-1127, 2000 
[28] W.J. Layton, F. Schieweck, and I. Yotov, Coupling fluid flow with porous media flow, SIAM J. Numer. Anal., 40(6), 2195-2218, 2003.

[29] M. Mu and J. Xu, A two-grid method of a mixed Stokes-Darcy model for coupling fluid flow with porous media flow, SIAM J. Numer. Anal., 45(5), 1801-1813, 2007.

[30] L.E. Payne, J.F. Rodrigues, and B. Straughan, Effect of anisotropic permeability on Darcy's law, Math. Meth. Appl. Sci., 24, 427-438, 2001.

[31] K.R. Rajagopal, M. Ruzicka, and A.R. Srinivasa, On the Oberbeck-Boussinesq approximation, Math. Mod. Meth. Appl. Sci., 6(8), 1157-1167, 1996.

[32] R. Rannacher, Finite element methods for the incompressible Navier-Stokes equations, in Fundamental Directions in Mathematical Fluid Mechanics, G.P. Galdi et al. (eds.), Advances in Mathematical Fluid Mechanics, Birkhäuser Verlag, Basel, 191-293, 2000.

[33] B. Rivière and I. Yotov, Locally conservative coupling of Stokes and Darcy flows, SIAM J. Numer. Anal., 42(5), 1959-1977, 2005.

[34] D. Vassilev and I Yotov, Coupling Stokes-Darcy flow with transport, SIAM J. Sci. Comput., 31(5), 3661-3684, 2009.

[35] S. Whitaker, The Method of Volume Averaging (Theory and Applications of Transport in Porous Media), Kluwer Academics Pub., Dordrecht 1999.

[36] A.M. Wilson, W. Sanford, F. Whitaker, and P. Smart, Spatial patterns of diagenesis during geothermal circulation in carbonate platforms, Amer. J. Sci., 301, 727-752, 2001.

[37] R. Wojnar, On nonlinear heat equations and diffusion in porous media, Reports on Math. Physics, 44(1-2), 291-300, 1999. 